\title{
The Aerogel Čerenkov Detector for the SHMS magnetic spectrometer in Hall C at Jefferson Lab
}

\author{
T. Horn ${ }^{\mathrm{a}, \mathrm{c}}$, H. Mkrtchyan ${ }^{\mathrm{b}}, \mathrm{S} . \mathrm{Ali}^{\mathrm{a}}$, A. Asaturyan ${ }^{\mathrm{b}}$, M. Carmignotto ${ }^{\mathrm{a}}$,

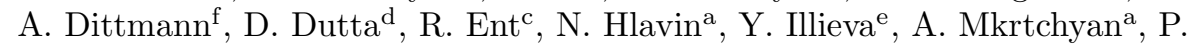 \\ Nadel-Turonskic ${ }^{\mathrm{c}}$, I. Pegg ${ }^{\mathrm{a}}$, A. Ramos ${ }^{\mathrm{g}}$, J. Reinhold ${ }^{\mathrm{g}}$, I. Sapkota ${ }^{\mathrm{a}}$,

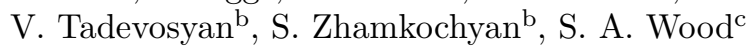 \\ ${ }^{a}$ The Catholic University of America, Washington, DC 20064, USA \\ ${ }^{b}$ A. I. Alikhanyan National Science Laboratory, Yerevan 0036, Armenia \\ ${ }^{c}$ Thomas Jefferson National Accelerator Facility, Newport News, Virginia 23606, USA \\ ${ }^{d}$ Mississippi State University, Mississippi State, Mississippi 39762, USA \\ ${ }^{e}$ University of South Carolina, Columbia, South Carolina 29208, USA \\ ${ }^{f}$ University of Illinois, Urbana-Champaign, Illinois, USA \\ ${ }^{g}$ Florida International University, University Park, Florida 33199, USA
}

\begin{abstract}
Hadronic reactions producing strange quarks such as the exclusive $p\left(e, e^{\prime} K^{+}\right) \Lambda$ and $p\left(e, e^{\prime} K^{+}\right) \Sigma^{0}$ reactions, or the semi-inclusive $p\left(e, e^{\prime} K^{+}\right) X$ reaction, play an important role in studies of hadron structure and the dynamics that bind the most basic elements of nuclear physics. The small-angle capability of the new Super High Momentum Spectrometer (SHMS) in Hall C, coupled with its high momentum reach - up to the anticipated $11-\mathrm{GeV}$ beam energy in Hall $\mathrm{C}$ - and coincidence capability with the well-understood High Momentum Spectrometer (HMS), will allow for probes of such hadron structure involving strangeness down to the smallest distance scales to date. To cleanly select the kaons, a threshold aerogel Cerenkov detector has been constructed for the SHMS. The detector consists of an aerogel tray followed by a diffusion box. Four trays for aerogel of nominal refractive indices of $n=1.030,1.020,1.015$ and 1.011 were constructed. The tray combination will allow for identification of kaons from 1 $\mathrm{GeV} / c$ up to $7.2 \mathrm{GeV} / c$, reaching $\sim 10^{-2}$ proton and $10^{-3}$ pion rejection, with kaon detection efficiency better than $95 \%$. The diffusion box of the detector is equipped with 14 five-inch diameter photomultiplier tubes. Its interior walls are covered with Gore diffusive reflector, which is superior to the commonly
\end{abstract}


used Millipore paper and improved the detector performance by $35 \%$. The inner surface of the two aerogel trays with higher refractive index is covered with Millipore paper, however, those two trays with lower aerogel refractive index are again covered with Gore diffusive reflector for higher performance. The measured mean number of photoelectrons in saturation is $\sim 12$ for $n=1.030, \sim 8$ for $n=1.020, \sim 10$ for $n=1.015$, and $\sim 5.5$ for $n=1.011$. The design details, the results of component characterization, and initial performance tests and optimization of the detector are presented.

Keywords: Silica aerogel, Threshold Cherenkov detector, particle identification, light yield, diffusive reflectors

\section{Introduction}

The 21st century holds great promise for reaching a new era for unlocking the mysteries of the inner quark-gluon structure of the atomic nucleus and the nucleons inside it as governed by the theory of strong interactions (QCD). The

5 experimental program at the upgraded $12 \mathrm{GeV}$ Jefferson Laboratory [1, 2, 3], currently nearing completion, will play an important role in this quest, and revolutionize the current understanding of the dynamics of the fundamental quarks and gluons that create the wide and varied structure of hadrons and nuclei.

10 Hall $\mathrm{C}$ with its heavily-shielded detector setup in a highly-focusing magnetic spectrometer with large momentum reach will be the optimal Hall for certain classes of deep exclusive and semi-inclusive measurements, and in particular those requiring high quality Rosenbluth (or longitudinal-transverse - L/T) cross section separations. The Hall $\mathrm{C}$ base experimental equipment consists of two magnetic spectrometers: the High Momentum Spectrometer (HMS) [4] and the Super High Momentum Spectrometer (SHMS) [5]. Depending on the specific requirements of the experiments, they can detect either negatively or positively charged particles by choosing the magnetic field and the trigger configuration. The HMS is designed to detect secondary products of reactions in the momen- 
tum range from 0.5 to $7.3 \mathrm{GeV} / c$, while the SHMS momentum range extends up to about $12 \mathrm{GeV} / c$. Both spectrometers are equipped with a pair of drift chambers, pairs of timing scintillator hodoscopes for trigger formation, and various detectors for particle identification purposes, including the aerogel Cerenkov detector. A schematic of the SHMS detector package is shown in Fig. 1(b).

Particle identification in the SHMS relies on time-of-flight measurements at lower momentum, an electromagnetic pre-shower and full shower calorimeter and Čerenkov detectors. A Čerenkov detector as filled with noble gases, and the calorimeter will be used for $e / \pi$ separation, while a separate Čerenkov detector filled with heavier gases such as $\mathrm{C}_{4} \mathrm{~F}_{8} \mathrm{O}$ will provide $\pi / K$ identification above $3.4 \mathrm{GeV} / c$. To complete the necessary PID capability of the SHMS necessary to successfully carry out the strangeness physics part of the Hall C program, the SHMS aerogel Čerenkov detector descriped in this paper was built to distinguish kaons from protons with momenta above $2.6 \mathrm{GeV} / c$.

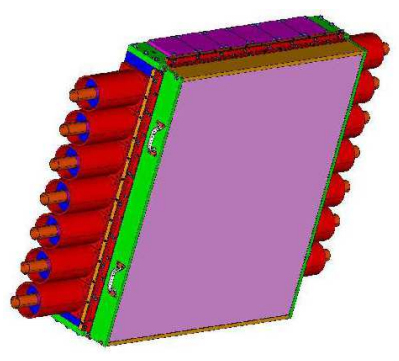

(a) Schematic drawing of the SHMS aerogel detector

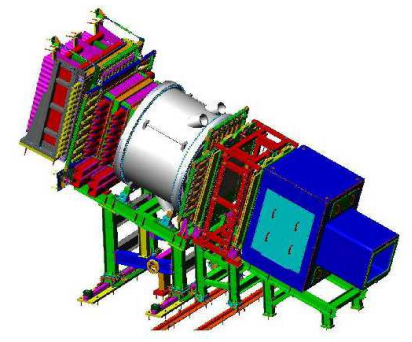

(b) Schematic side-view of the SHMS detector package

Figure 1: (Color online) Schematic drawings of the SHMS aerogel detector (left, a) and its placement in the SHMS detector hut (right, b). The detector stack of the SHMS consists, right to left, of a gas Čerenkov, a pair of drift chambers, a pair of $X-Y$ segmented scintillator, a gas Cerenkov (gray cylinder), two possible slots for the aerogel detector, each of $30 \mathrm{~cm}$ depth, a second $X-Y$ segmented scintillator/quartz bar hodoscope, and an electromagnetic calorimeter.

Meson production experiments such as the "Scaling Study of the L-T Separated Pion Electroproduction Cross Section at 11 GeV" (E12-07-105) [6] and "Studies of the L-T Separated Kaon Electroproduction Cross Section from 5-11 
GeV" (E12-09-011) 7], will study the potential for 3D hadron imaging studies and may also allow for form factor extractions in a kinematic regime where the signatures of QCD are quantitatively revealed [8, 9]. These experiments will provide high-quality cross section data, requiring detection of scattered mesons with momenta approaching $10 \mathrm{GeV} / c$, for which the SHMS in Hall $\mathrm{C}$ is exceptionally well suited. However, additional instrumentation for $K^{+} / p$ particle identification is needed for the kaon measurement, which forms an essential part of this program. The " $P_{T}$ in SIDIS" experiment (E12-09-017) [10] will

45 map the transverse momentum dependence of semi-inclusive electroproduction of charged mesons from both proton and deuteron targets. In this experiment it will be beneficial to also separate kaons from protons. The "Measurement of $R=\sigma_{L} / \sigma_{T}$ in SIDIS" (E12-06-104) [11] will study the inclusive-exclusive connection in meson electroproduction, where duality has recently been shown to be valid and will be essential in understanding flavor decomposition in semiinclusive deep inelastic scattering at $12 \mathrm{GeV}$. With $K^{+} / p$ separation this measurement can access the additional flavors of strange systems. The "Pion Transparency" experiment (E12-06-107) [12] will map the transparency of the nuclear medium to pions and measure the approach to the QCD-calculable, or point-like, regime using the $Q^{2}$ dependence of the ratio of cross sections from nucleon and nuclear targets. Kaon transparency measurements may contribute additional information to our understanding of this approach. The general requirement for these experiments is a high detection efficiency for kaons in the SHMS and the capability to separate protons from kaons. The experiments plan to run with an electron beam with energy of up to $11 \mathrm{GeV}$ and beam currents between a few and $80 \mu \mathrm{A}$ hitting liquid hydrogen or deuterium target cells with lengths about $10 \mathrm{~cm}$, or selected solid targets, yielding particle rates in the SHMS detector stack of up to $2 \mathrm{MHz}$. To take full advantage of the existing SHMS standard detector configuration without compromising its performance, the additional aerogel Čerenkov detector system was designed with the following specifications: 
- to have a sensitive area of at least $90 \times 60 \mathrm{~cm}^{2}$ with a thickness of radiator up to $9 \mathrm{~cm}$.

- to fit into a small slot of about $30 \mathrm{~cm}$ between the heavy gas Čerenkov detector and the electromagnetic calorimeter;

- to minimize the material in the particle path to keep the amount of multiple scattering and $\delta$-electrons small;

- to allow easy exchange of the aerogel material to match the required kaon momentum range;

75

- to provide acceptable time resolution and a high-rate capability.

Of the approved experiments, three (E12-06-104, E12-09-017, and E12-07-105) only require kaon particle identification (PID) in the $2.7-5 \mathrm{GeV} / c$ momentum range. Higher momenta are relevant for the kaon factorization experiment (E1209-011), up to $7.2 \mathrm{GeV} / \mathrm{c}$, and the pion transparency measurement, where kaon identification up to this momentum would allow extraction of kaon transparencies up to $Q^{2} \sim 7(\mathrm{GeV} / c)^{2}$. To take into account this range of experimental requirements, covering from about 2 to above $7 \mathrm{GeV} / c$ momentum, the refractive indices characteristic of aerogel material are well suited for this purpose.

To maintain good $K^{+} / p$ separation over this full kinematic range, we chose a range of aerogel materials, with nominal refractive indices of $n=1.030$ (SP-30), $n=1.020$ (SP-20), $n=1.015$ (SP-15), and $n=1.011$ (SP-11). The threshold momenta (in $\mathrm{GeV} / c$ ) for muons, pions, kaons and protons in these types of aerogel are listed in Table 1. Up to about $4 \mathrm{GeV} / c, K^{+} / p$ separation is achieved with aerogel with a refractive index of $n=1.030$. Beyond, the lower three refractive indices provide excellent $K^{+} / p$ separation in the momentum range between 4 and $\sim 7 \mathrm{GeV} / c$. 
Table 1: Threshold momenta $\mathrm{P}_{T h}$ for Čerenkov radiation for charged muons, pions, kaons, and protons in aerogel of four refractive indices ranging from $n=1.011$ to 1.030 .

\begin{tabular}{||l|c|c|c|c|}
\hline Particle & $\begin{array}{c}\mathrm{P}_{T h} \\
n=1.030\end{array}$ & $\begin{array}{c}\mathrm{P}_{T h} \\
n=1.020\end{array}$ & $\begin{array}{c}\mathrm{P}_{T h} \\
n=1.015\end{array}$ & $\begin{array}{c}\mathrm{P}_{T h} \\
n=1.011\end{array}$ \\
\hline$\mu$ & 0.428 & 0.526 & 0.608 & 0.711 \\
$\pi$ & 0.565 & 0.692 & 0.803 & 0.935 \\
$K$ & 2.000 & 2.453 & 2.840 & 3.315 \\
$p$ & 3.802 & 4.667 & 5.379 & 6.307 \\
\hline \hline
\end{tabular}

This paper is organized as follows. Details regarding the design of the detector, such as the diffusion box, tray construction, and a short description of the detector components are given in section 2, Section 3 presents the results

of the aerogel optical properties based on a randomly selected sample of 5-15\%

of the aerogel tiles of each refractive index used in the detector. Variations in refractive index, dimensions, and surface shape of the tiles are also briefly described. The construction of a single counter (prototype) and tests performed with it are described in section 3 The description of the methods used to study the photomultiplier tubes, the results of these studies and the principles guiding selection of the PMTs for the detector are given in section 4. The results of initial performance tests of the aerogel Cerenkov detector and a comparison to simulations are presented in section 5. Finally, the results are summarized in section 6 .

\section{Design overview}

The SHMS aerogel detector is installed in the detector hut of the SHMS between the heavy gas Čerenkov and the second hodoscope plane (see Fig. 11). The detector consists of two main components: a tray which holds the aerogel material, and a light diffusion box with photomultiplier tubes (PMTs) for light readout, as illustrated in Fig. 2. To cover the required kaon identification momentum range of up to $7.2 \mathrm{GeV} / c$, four identical trays for aerogel of nominal 
refractive indices of 1.030, 1.020, 1.015 and 1.011 were constructed. The design allows for easy detector assembly and replacement of the aerogel trays.

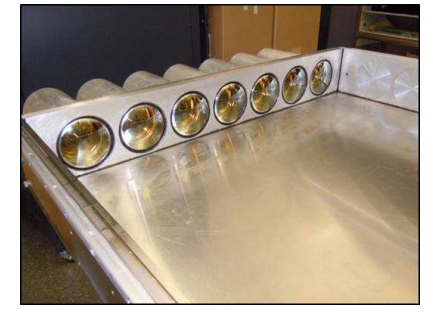

(a) Assembled diffusion box

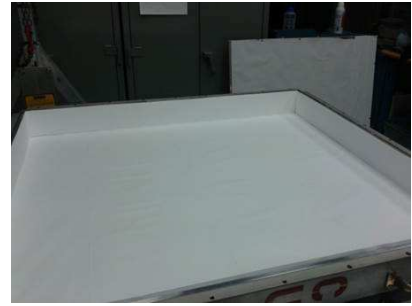

(b) Aerogel tray

Figure 2: (Color online) Diffusion box (left, a) and aerogel tray (right, b) of the SHMS aerogel detector. Both are covered with a diffuse reflector material.

An active area of $60 \mathrm{~cm}$ (width) and $90 \mathrm{~cm}$ (height) fully satisfies the needs of the approved experiments [6, 7, 10, 11, 12] requiring kaon identification, which will all use targets with lengths of about $10 \mathrm{~cm}$. The momentum range (around the chosen spectrometer central momentum) and angular phase space accepted by the SHMS for an assumed $10 \mathrm{~cm}$ target length corresponds to en assemble of particle rays in the SHMS detector hut, at the location of the aerogel detector, of less than this active area. However, the detector was designed and built with a total area of $110 \times 100 \mathrm{~cm}^{2}$ to in principle also allow coverage of the full momentum and angular range of the spectrometer for a 40-cm long target.

Using 5-inch diameter PMTs mounted on the vertical sides of the diffusion box and up to $9 \mathrm{~cm}$ aerogel thickness in the trays, the total depth of the detector 125 is $24.5 \mathrm{~cm}$ along the optical axis of the SHMS. Such a compact detector is well suited for the limited space available, up to $30 \mathrm{~cm}$, in the SHMS detector stack.

Prior to construction, the performance requirements of the detectors were studied and optimized using a dedicated Monte Carlo code [13]. The baseline design for this optimization assumed a $110 \times 100 \times 24.5 \mathrm{~cm}^{3}$ detector box covered from the inside with a diffuse reflector of at least $96 \%$ reflectivity, and with 5 -inch diameter PMTs of $20 \%$ quantum efficiency mounted on each long side of the diffusion box. This design concept follows earlier proven aerogel detector 
designs [14, 15, 16]. Since the photon detection probability is directly proportional to the fraction of the inner detector surface covered by the photo-cathode windows of the PMTs [17, 18], the mechanically allowable maximum number of seven PMTs on each side was assumed in these studies. The simulations showed that the number of photo-electrons $N_{p e}$ as measured by all PMTs summed is uniform to within $10 \%$ over the full active area of the detector, with this twosided readout.

The diffusion box is made of the aluminum alloy 6061-T6. The side panels are constructed of $\sim 2.5 \mathrm{~cm}$ (1-inch) plates. The back cover is $\sim 1.6 \mathrm{~mm}(1 / 16$ inch) thick. The inner dimensions of the box are $\sim 103 \times 113 \times 17.3 \mathrm{~cm}^{3}(40.5 " \times$ 44.5 " $\times 6.82$ "). To optimize light collection the inner surface of the diffusion box is lined with either $3 \mathrm{~mm}$ (covering $\sim 60 \%$ of the surface) or $1 \mathrm{~mm}$ (remaining $145 \sim 40 \%$ of the surface) thick GORE reflector material [19]. This material has a reflectivity of about $99 \%$ over the entire spectrum. Further discussion of this material and its effect on light collection can be found in section 5.3 .

The light collection is handled by 5 -inch diameter photomultiplier tubes. The $5.56 "(14.1 \mathrm{~cm})$ diameter cylindrical housings holding the PMTs are mounted upon 14 waterjet cut circular openings on the left and right (long) sides of the diffusion box, with minimum spacing of $14.92 \mathrm{~cm}$ (5.875") between the centers. The PMTs are sealed into their housing using a light-tight synthetic rubber material (Momentive RTV103 Black Silicone Sealant) and the whole assembly is sealed light-tight. The mechanical design includes six openings on the top of 155 the diffusion box, presently covered with blanks, that can be used to increase the signal output from the detector by about $30 \%$, if needed.

The magnetic shielding for the PMTs consists of $13.5 \mathrm{~cm}$ (5.316") diameter $\mu$-metal cylinders, which were constructed to end abreast with the PMT window. The construction also features bucking coils that can be installed on the PMTs, if excessive residual magnetic fields appear to be present in the SHMS hut. The selection criteria for the PMTs and studies on efficiency of the magnetic shielding including the bucking coil studies, are presented in section 4.3 ,

The aerogel trays are of the same transverse size as the diffusion box but 
$11.3 \mathrm{~cm}(4.45 ")$ deep. The front cover of the trays is made of a $5 \mathrm{~mm}$ thick honeycomb panel with effective Aluminum thickness to $\sim 1.3 \mathrm{~mm}(0.050 ")$. The inner surface of the SP-30 and SP-20 aerogel trays is covered with $0.45 \mu \mathrm{m}$ thick Millipore paper Membrane GSWP-0010 (Millipore) of reflectivity of about 96\% [20]. Though Millipore is difficult to handle, its chemical inertness makes it superior to reflective paints. For the two lower refractive index trays (SP-15 and SP-11), in order to optimize light collection, we used $1 \mathrm{~mm}$ thick Gore diffusive reflector material (DRP-1.0-12x30-PSA) with reflectivity of about $99 \%$.

For the Čerenkov radiator high transparency aerogels were used. The higher two of the refractive indices (SP-30 and SP-20) were originally manufactured by Matsushita Electric Works, Ltd. The lower two indices (SP-15 and SP-11) were manufactured by Japanese Fine Ceramics Center. These tiles have dimensions of approximately $11 \mathrm{~cm}$ by $11 \mathrm{~cm}$ by $1 \mathrm{~cm}$. They feature a waterproof coating that make them hydrophobic [21, 22]. This removes the need for baking (which in fact would destroy the coating). Detailed studies of the aerogel characteristics are presented in section 3 .

The trays were filled with aerogel tiles layer by layer. In each layer the tiles were layed down flat and arranged in a brick pattern to minimize holes in the radiator. To fill gaps of less than the size of a full tile at the edges of the tray the aerogel material was cut using a diamond coated saw or razor depending on the refractive index of the material. The aerogel radiator is on average $\sim 9 \mathrm{~cm}$ thick (8 layers). The SP-30, SP-20 and SP-15 aerogel trays were filled over their entire $110 \mathrm{~cm}$ x $100 \mathrm{~cm}$ area. The SP-11 aerogel tray radiator covers only the active area of $90 \mathrm{~cm}$ x $60 \mathrm{~cm}$ required by the experiments. An inner frame has been designed to arrange the aerogel tiles inside the active area of this tray (see Fig. 31). The sides of this inner frame are made of carbon fiber square tubes. This assembly allows future X-Y repositioning of the inner frame inside the tray.

To protect the aerogel radiator from severe damage in case of accidental flipping over of a tray during installation, a net of thin stainless steel wires is installed in close proximity to the aerogel surface. This is a technique previously tested in aerogel detectors at JLab [16]. The wires form an interweaving grid 
by running between stainless steel screws on the sides of the box. Small springs attached to the ends of wires provide necessary tension.

An aerogel tray attaches to the diffusion box by means of bolting through flanges surrounding both boxes. A round O-ring running in a shallow groove along the diffusion box sides ensures a light tight connection. The entire detector is designed so that it can be removed from the sliding detector stand that positions the detector into the SHMS detector stack.
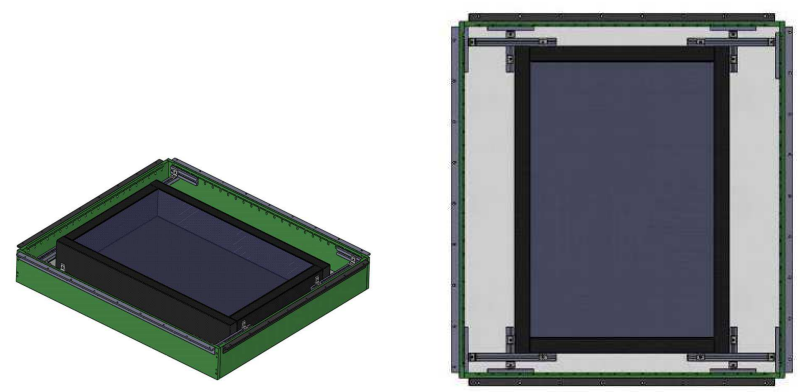

Figure 3: (Color online) Schematic drawing of the SP-11 aerogel tray with inner frame covering the active area of $90 \mathrm{~cm} \times 60 \mathrm{~cm}$.

The SHMS aerogel detector was designed considering the optimization of the amount of material in the particles's path in the spectrometer. The amount of material across the sensitive area of the detector was minimized to reduce effects of multiple scattering and production of $\delta$-electrons, but with the optimal thickness of radiator required to achieve higher PID efficiency with the detector. Table 2 summarizes the effective thickness of materials in the path of particles transversing the SHMS aerogel detector, with the respective radiation length of these materials.

\section{Aerogel characterization}

For the assembly of the trays with nominal refractive indices $n=1.030$ and 1.020, aerogel tiles from an earlier experiment [23, 24] were used, where they had 
Table 2: List of materials in the path of particles traversing the SHMS aerogel detector, with their respective radiation length.

\begin{tabular}{|c|c|c|c|c|}
\hline Component & Material & $\begin{array}{c}\text { Thickness } \\
(\mathrm{cm})\end{array}$ & $\begin{array}{c}\text { Density } \\
\left(\mathrm{g} / \mathrm{cm}^{3}\right)\end{array}$ & $\begin{array}{c}\text { Radiation Length } \\
\left(\mathrm{g} / \mathrm{cm}^{2}\right)\end{array}$ \\
\hline Window & $\mathrm{Al}$ & 0.13 & 2.7 & 24.01 \\
Aerogel & $\mathrm{SiO}_{2}$ & 9.0 & 0.2 & 44.054 \\
Air gap & $\mathrm{Air}$ & 17.1 & 0.00121 & 36.66 \\
Window & $\mathrm{Al}$ & 0.16 & 2.7 & 24.01 \\
\hline
\end{tabular}

been exposed to a relatively harsh radiation environment. The trays with nom-

inal refractive indices $n=1.015$ and 1.011 contain aerogel tiles acquired directly from the manufacturer albeit in several batches. To check for possible degradation of the previously used tiles and dependence on the manufacturing process the properties and uniformity of the aerogel were carefully analyzed prior to the assembly of the detector. For each nominal refractive index a sample of $5-15 \%$ of all the tiles was randomly selected and tested. In the following subsections, we report the studies of the most important properties of the aerogel for the construction of the detector: refractive index, tile dimensions, light yield, light transmittance and absorption, and hydrophobicity.

Several features of the detector like the selection of reflector material or thickness of the aerogel radiator also required careful studies before the final assembly. A prototype counter was constructed to study and optimize these features of the actual detector. The prototype was a combination of a small aerogel tray (which can be filled with up to 14 aerogel tiles) and a diffusion box viewed by a single PMT. The internal surface of the prototype was covered with

230 Millipore material or aluminized Mylar as reflective material. The prototype is shown in Fig. 4 .

This single-PMT prototype allowed studies of, e.g., different reflective materials covering the walls of the detector, comparative light yield of the different aerogel refractive indices, and optimization of performance with PMTs. After 35 the conclusion of the tests, the construction of the entire array of the actual 


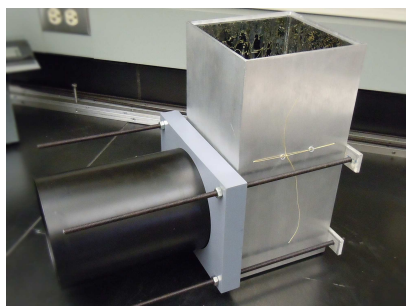

(a) Prototype picture

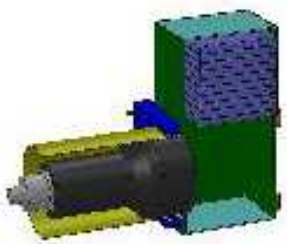

(b) Prototype drawing

Figure 4: (Color online) Prototype of the aerogel detector built for studies of the characteristics of the detector. Left (a) the prototype with the top cover of the aerogel box removed. Right (b) a schematic drawing of the prototype showing the position of the PMT (in gray) and aerogel tiles (blue) stacked inside the prototype.

detector proceeded without significant changes from the prototype.

\subsection{Refractive index}

The velocity threshold for production of Čerenkov radiation inside a material is given by $v_{t}=c / n$, where $c$ is the speed of light in vacuum and $n$ is the refractive index of the material. The momentum threshold of a particle associated with $v_{t}$ is given by $p_{t}=m / \sqrt{\left(n^{2}-1\right)}$, where $m$ is the mass of the particle.

In the SHMS Aerogel Cerenkov Detector, the refractive index of each tray of aerogel defines the momentum range that the detector will allow for distinguishing between kaons and protons in the PID system of the SHMS. Fig. 5 shows the dependence of the momentum threshold, $p_{t}$, for radiation of Cerenkov light for pion, kaon and proton as a function of the refractive index of the aerogel.

Measurements of the refractive index of tiles were performed with a technique based on Snell's Law. A similar method was used as in Ref. [25], it consists of measuring the refraction of a beam of light (red, wavelength of $670 \mathrm{~nm}$ ) incident on one corner of an aerogel tile. As illustrated in Fig. 6, the beam of light is incident on the tile at an angle $\alpha$ with respect to the normal to the surface of its corner. The section of the beam of light that goes through the analyzed tile is refracted and hits a screen placed at a distance $L$ from the tile. Refraction bends the incident beam of light in the aerogel material, so that it is separated from 


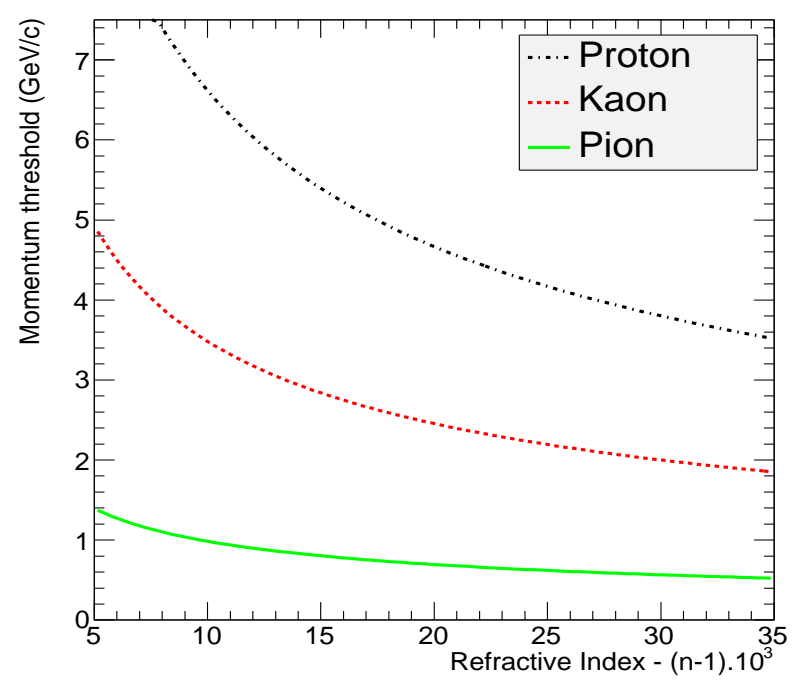

Figure 5: (Color online) Momentum threshold for proton, pion and kaon above which Čerenkov light is radiated in aerogel, according to the aerogel refractive index. On this horizontal scale, SP-11 (SP-15, SP-20, SP-30) corresponds to 11 (15, 20, 30, respectively). 
the non-refracted part of the beam that does not go through the aerogel (direct beam) by a distance $x$ on the screen. From these variables and the angle $\beta$ between two sides of the aerogel tile (90 degrees in our case), one can determine the refractive index $n$ of the tile using,

$$
\frac{n}{n_{\text {air }}}=\sqrt{\frac{\sin ^{2}(\alpha)+\sin ^{2}(\gamma)}{\sin ^{2}(\beta)}+2 \frac{\sin (\alpha) \sin (\gamma)}{\tan (\beta) \cdot \sin (\beta)}},
$$

where

$$
\gamma=\tan ^{-1}\left(\frac{x}{L}\right)-\alpha+\beta
$$

and $n_{\text {air }}$ is the refractive index of the air surrounding the aerogel tile. Based on the temperature, atmospheric pressure and relative humidity of our laboratory, we considered $\left(n_{\text {air }}-1\right) \times 10^{5}=26.5 \pm 0.5$, according to [26].

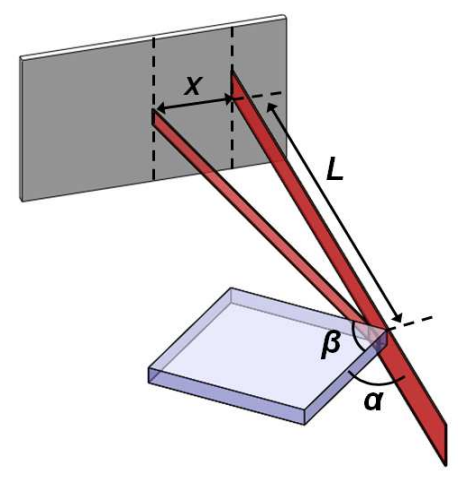

Figure 6: (Color online) Schematic of the setup used to measure the refractive index of aerogel tiles. A beam of light is incident over one edge of the tile. Refraction of the outgoing light is used to calculate its refractive index based on Snell's Law.

Figure 7 shows the statistical distribution of the refractive index measured for a randomly selected sample of the aerogel tiles used in the assembly of the trays. The dominant source of systematic uncertainties in this setup is the measurement of the angles $\alpha$ and $\beta$. The angles can have an offset of up to 0.8 degrees resulting in a systematic error of $\pm 0.72 \times 10^{-3}$ on the refractive index. 


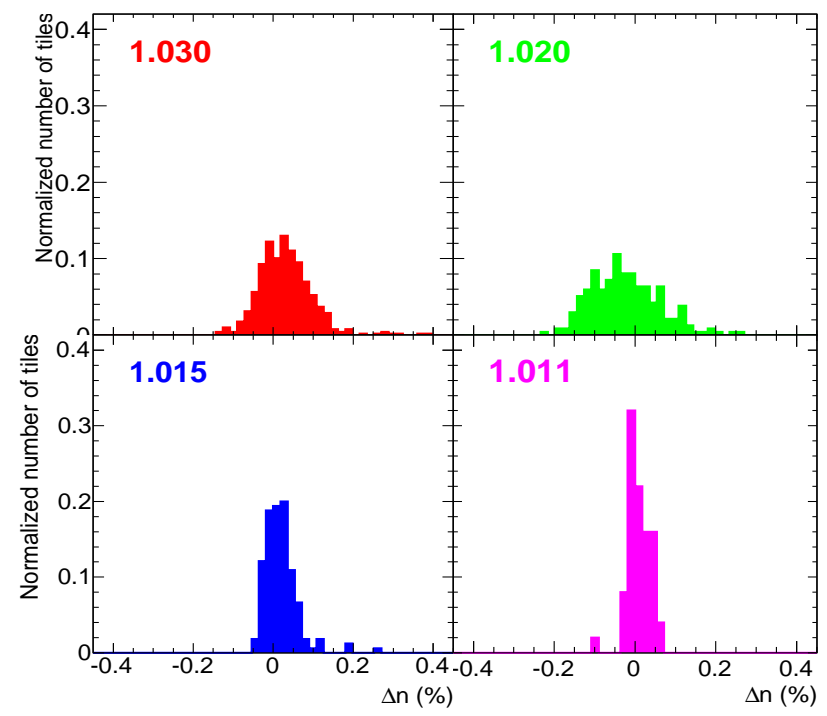

Figure 7: (Color online) The deviation of the refractive index of the individual tiles to the nominal refractive index of each tray. The distributions were measured for a sample of $5-10 \%$ of the tiles used for the assembly of the four trays of the detector. 
Table 3: Aerogel refractive index measured for a sample of the tiles used on the construction of each tray. The refractive index of aerogel was calculated using equation 1 assuming $n_{\text {air }}=1.000265$.

\begin{tabular}{cc}
\hline Tray & Refractive index \\
\hline 1.030 & $1.0303 \pm 0.0007$ \\
1.020 & $1.0198 \pm 0.0009$ \\
1.015 & $1.0152 \pm 0.0004$ \\
1.011 & $1.0111 \pm 0.0003$ \\
\hline
\end{tabular}

The SP-30 tiles of nominal index $n=1.030$ had a measured mean value of $n=1.0303$ and a standard deviation of 0.0007 . For the SP-20 tiles of nominal index $n=1.020$, the measured mean value was $n=1.0198$ with a standard deviation of 0.0009 . The SP-15 tiles had a mean value of $n=1.0152$ and a standard deviation of 0.0004. Finally, for the SP-11 tiles, the mean value was $n=1.0111$ with a standard deviation of 0.0003 . The uncertainty of the refractive index of air is a decade less, thus the systematic errors are dominated by the statistical variation of the refractive index from tile to tile. The refractive indices and uncertainties are summarized in Table 3

We do note that the manufacturer of the aerogel reported the refractive index of the tiles used in the tray SP-15 and SP-11 as $n=1.01495 \pm 0.00020$ and $n=1.01066 \pm 0.00010$, respectively. The ratio of these numbers and the mean values we measured is about 1.0003 , similar to $n_{\text {air }}$ and, for this reason, we believe the manufacturer may have calculated their values of refractive index with respect to air, whereas we calculated the refractive index of aerogel relative to vacuum.

\subsection{Dimensions of the aerogel tiles}

The thickness and the width of a sample of aerogel tiles were measured. For the measurement of the aerogel tile thickness each aerogel tile was placed 275 in between two aluminum plates. Using a caliper, we measured the thickness of each tile from the distance between these two aluminum plates. Similar, a 
caliper was used to measure the lateral width of each tile of the selected sample of aerogel. The distance of two opposite sides of the tiles was measured in several points along the dimension of each aerogel tile and an average over these measurements was considered as the tile width. Table 4 summarizes the dimensions of the aerogel tiles used in the assembly of the SHMS Aerogel Cerenkov Detector.

Table 4: Dimensions of (a sample of) aerogel tiles used on the construction of the detector's trays.

\begin{tabular}{ccc}
\hline Tray & Width $(\mathrm{mm})$ & Thickness $(\mathrm{mm})$ \\
\hline 1.030 & $113.10 \pm 0.40$ & $11.583 \pm 0.067$ \\
1.020 & $110.82 \pm 0.59$ & $11.42 \pm 0.33$ \\
1.015 & $111.83 \pm 0.22$ & $11.10 \pm 0.15$ \\
1.011 & $112.28 \pm 0.35$ & $10.93 \pm 0.10$ \\
\hline \hline
\end{tabular}

\subsection{Light yield - Čerenkov radiation}

The basic physical principle behind the particle identification with the SHMS Aerogel Čerenkov detector is the emission of Čerenkov radiation in the aerogel when the speed of a particle going through the detector is larger than the phase velocity of the electromagnetic fields inside this material, i.e. $v>c / n$. Combined with knowledge on the momentum of the detected particles as provided by the magneto-optics analysis of the events in the spectrometer, the production of Čerenkov radiation is then used as a mass analyzer.

Based on classical electrodynamics and the quantization of light, one can estimate the energy $(E)$ spectrum and the number of photons $(N)$ produced per unit track length $(x)$ of a particle with charge ze and per unit energy interval of the photons according to Ref. [27] as

$$
\frac{d^{2} N}{d E d x}=\frac{\alpha z^{2}}{\hbar c}\left(1-\frac{1}{\beta^{2} n^{2}(E)}\right),
$$


or, equivalently,

$$
\frac{d^{2} N}{d x d \lambda}=\frac{2 \pi \alpha z^{2}}{\lambda^{2}}\left(1-\frac{1}{\beta^{2} n^{2}(\lambda)}\right),
$$

where $\alpha$ is the fine structure constant, $\hbar$ is the Planck's constant, $c$ is the speed of light, and $\beta$ is the fractional velocity of the incoming particle $(\beta=v / c)$. $n$ is the refractive index of aerogel and a function of photon energy (or wavelength $\lambda)$.

Figure 8 shows an estimate of the total light yield expected for the different nominal refractive indices of aerogel, for a thickness of $10 \mathrm{~cm}$. This estimate is based on the method discussed in Ref. [13], where effects such as light absorption in the components of the detector and quantum efficiency of the PMTs were taken into account. Note that this estimate is done for the baseline design aerogel Čerenkov detector as described in section 2 . 


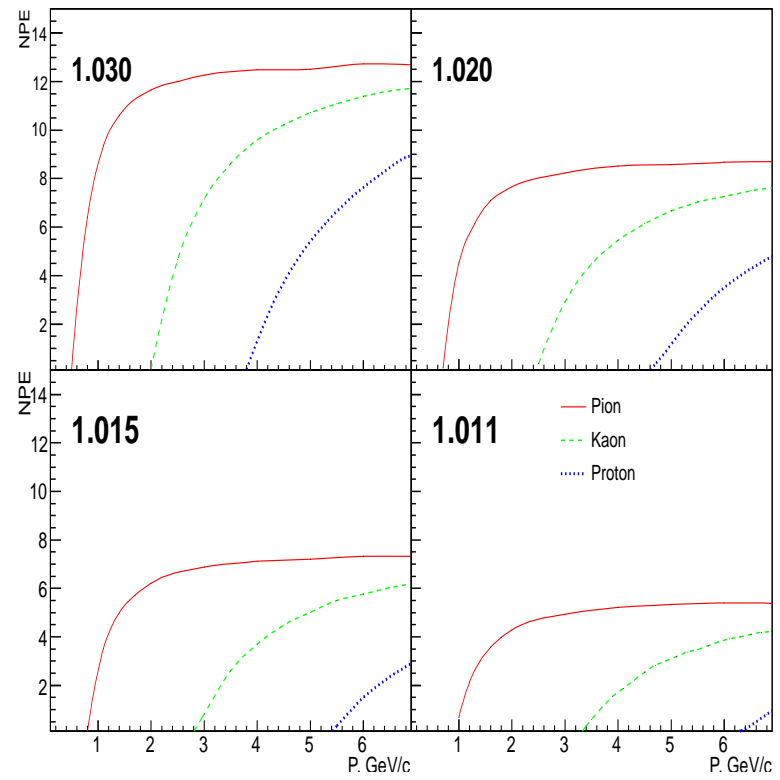

Figure 8: (Color online) Estimated mean number of photoelectrons (NPE) to be measured by the "baseline design" SHMS Aerogel Čerenkov Detector for the trays with different refractive index. This calculation was done following the model proposed by the authors of Ref. 13], for the simulation of an aerogel Čerenkov similar to our detector.

The calculated number of photo-electrons in saturation (for $\beta=v / c \sim 1$ particles), is $\sim 12$ for aerogel with nominal refractive index $n=1.030$, and $\sim 4.6$ for $n=1.011$. Due to their relatively lower velocity $(\beta<1)$ kaons and protons in the same momentum range are far from saturation. Figure 8 shows the calculated numbers of photo-electrons for kaons and protons over the entire momentum range required by the experiments in section 1

To check the relative optical quality of the aerogel material we have measured the light yield generated by cosmic muons using the single counter (prototype) described above. Fig. 9 shows the number of photoelectrons as a function of aerogel thickness (or number of layers). We measure a nearly linear dependence of the number of photoelectrons on the aerogel thickness up to $\sim 9 \mathrm{~cm}$ (8 layers), as illustrated by the dotted line in Fig. 9, although some saturation may set in 
around 6 layers or so. For aerogel thicknesses of more than $11 \mathrm{~cm}$ (10 layers) the light yield clearly saturates as shown by the second-order polynomial fit (solid 320 curve) - some of the produced Čerenkov light gets reabsorbed.

To check for possible degradation of previously used aerogel material, we compared the results of measurements using 10 tiles $(\sim 11 \mathrm{~cm})$ of new and previously used $n=1.030$ aerogel. Both aerogel material having seen the radiation dose of the previous experiments and new aerogel material are indistinguishable. These tests suggest, within the uncertainty of the measurements, that the previously used aerogel tiles have a light yield similar to the new tiles and do not show significant aging or radiation degradation. Our result is consistent with the investigations of radiation damage of aerogel material in Ref. [28, 29]. There, aerogel tiles were exposed to very intense $\gamma$-radiation from a ${ }^{60} \mathrm{Co}$ source, and to proton and neutron high intensity beams. Transmittance, clarity factor and refractive index of the aerogel tiles were measured before and after irradiation and no detectable degradation of the optical parameters was observed up to doses of $\sim 10$ Mrad. Ref. [29] also showed that cracks in the aerogel tiles do not make a noticeable difference in the light yield.

The same selection criteria on optical properties and geometrical conditions (no chipped edges) were thus used for all tiles in the assembly of the SHMS Aerogel Čerenkov Detector. 


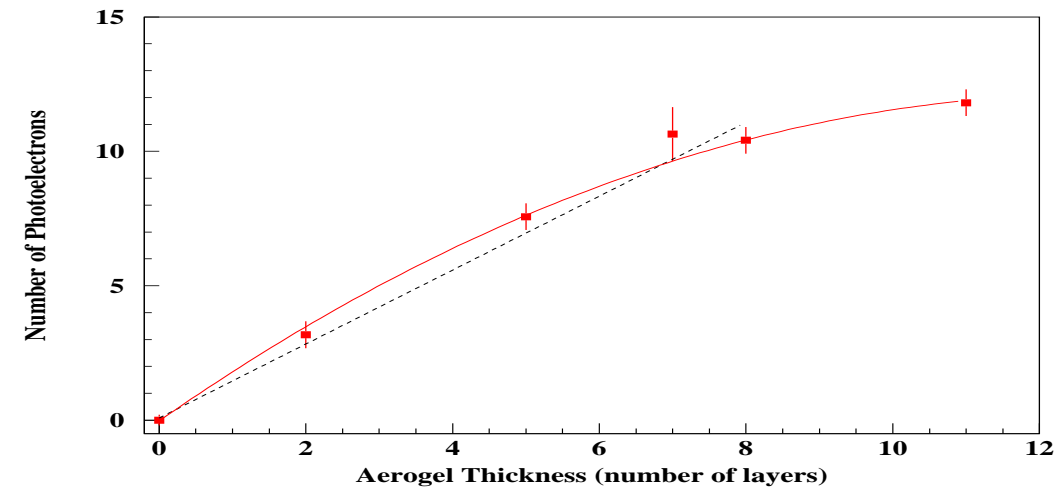

Figure 9: (Color online) The number of photoelectrons versus the aerogel radiator thickness (varying the number of layers) generated by cosmic muons in the single counter prototype. The dotted line represents a linear fit to the experimental data up to an aerogel thickness of 8 layers $(\sim 9 \mathrm{~cm})$. The solid curve shows a second-order polynomial fit to all data set up to a thickness of 11 layers $(\sim 12 \mathrm{~cm})$. Aerogel tiles with refractive index $n=1.030$ were used.

\subsection{Aerogel Light transmittance}

To quantify the transmittance of light through aerogel, a Perkin/Elmer LAMBDA $750 \mathrm{UV} /$ Vis/NIR Spectrophotometer was used to measure the light transmittance for a randomly selected sample of aerogel tiles for each nominal refractive index. In this spectrometer, a beam of light with tunable wavelength, $\lambda$, is split into two beams. The first beam goes directly to the light sensor for the measurement of the reference light intensity, while the second beam goes through one aerogel tile. The thickness of aerogel that the second beam goes through is shown in Table 4 .

The light transmittance of an aerogel tile is measured from the intensity of light that does not scatter or that is not absorbed by the aerogel, measured relative to the intensity of the reference beam. 


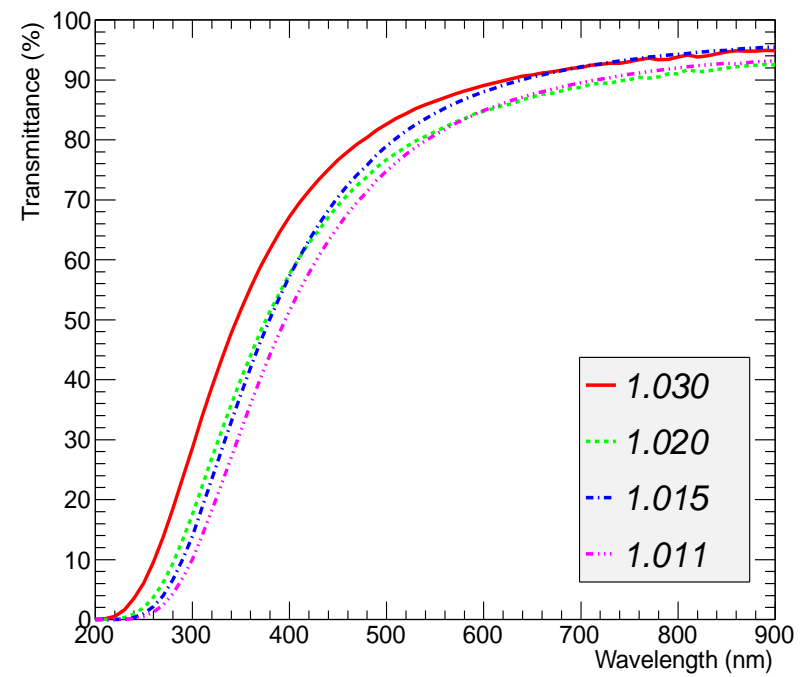

Figure 10: (Color online) Mean value of the light transmittance of aerogel measured over a sample of $\sim 10 \%$ of the tiles used on the construction of the detector. The mean thickness of the tiles for each refractive index is shown in Table 4

Fig. 10 shows the mean values of the aerogel's transmittance for wavelengths ranging from $200 \mathrm{~nm}$ to $900 \mathrm{~nm}$ as analyzed by the spectrometer. SP-30 tiles have a higher transmittance than SP-20, SP-15 and SP-11 tiles for wavelengths less than $600 \mathrm{~nm}$ including the region of interest for the SHMS Aerogel Čerenkov Detector. In this region the transmittance of SP-20 and SP-15 is similar and SP-11 has the lowest transmittance. For wavelengths greater than $600 \mathrm{~nm}$ the transmittance of the SP-15 tiles becomes similar to that of SP-30 and even exceeds it for wavelengths of greater than $700 \mathrm{~nm}$.

The transmittances for different tiles of the same refractive index are tightly clustered. We found that the statistical fluctuation of light transmittance for the different tiles with the same refractive index is less than $4 \%$. The systematic uncertainty of the measurements is $\pm 0.1 \%$. 


\subsection{Aerogel light absorption}

Aerogel Čerenkov detectors can exhibit light loss due to scattering and absorption in the aerogel material, as determined by the scattering and absorption legths.

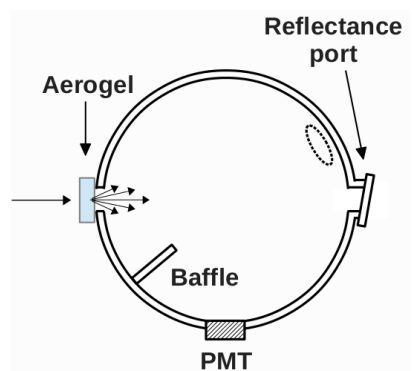

(a) Transmittance mode.

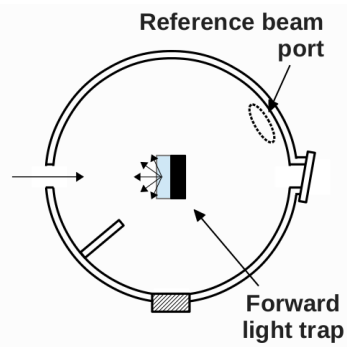

(b) Backscattering mode with forward light trap

Figure 11: (Color online) The integrating sphere equipped for transmittance and backscattering measurements.

We measured the light absorption in the aerogel tiles using a Perkin-Elmer Lambda 950 spectrophotometer with a $150 \mathrm{~mm} \mathrm{PbS}$ integrating sphere. The latter is covered with Spectralon, a material with high reflectivity. The integrating sphere can be operated in transmittance or backscattering mode as

illustrated in Fig. 11] The transmittance mode measures the light intensity that is either transmitted directly through the aerogel or scattered in the forward direction. The backscattering mode takes advantage of a forward light trap, which effectively allows one to measure only backscattered light from the aerogel. To determine the light absorption in aerogel, measurements were taken with the transmittance mode, with the sample outside the integrating sphere, and with the backscattering mode, where the sample was installed on a center mount with forward light trap inside the sphere. To take into account possible backgrounds we also took data without the aerogel installed in backscattering mode. Fig. 12 shows the measured light intensity of aerogel of refractive index $n=1.030$ in transmittance and backscattering modes. 


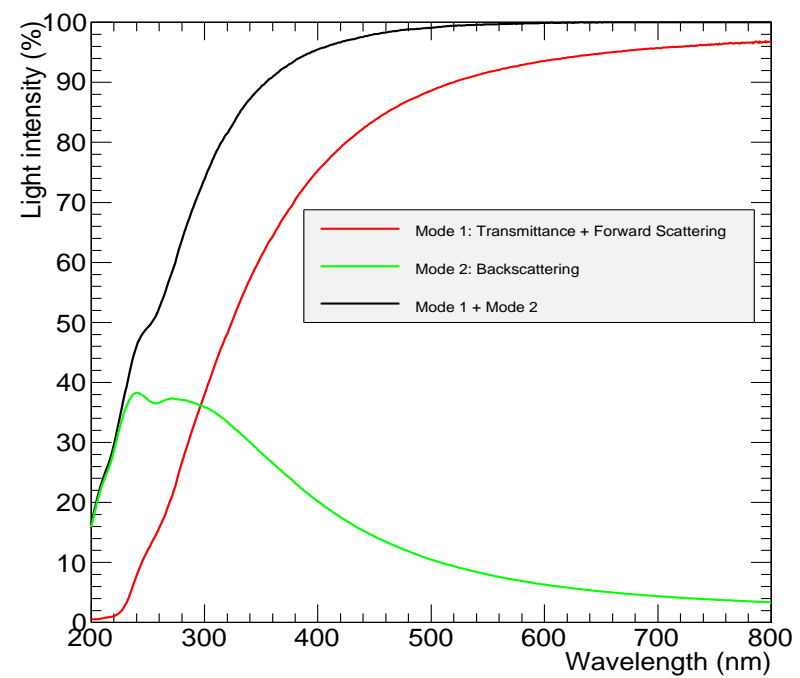

Figure 12: (Color online) Typical measured transmittance and backscattering for a range of wavelengths ranging from 200 to $800 \mathrm{~nm}$ in steps of $1 \mathrm{~nm}$.

The sum of the light intensity - $I(\lambda)$ - measured in transmittance and backscattering modes are related to the absorption length of the aerogel through the Beer-Lambert law,

$$
I(\lambda)=e^{\left.-t / \Lambda_{a b s}(\lambda)\right)},
$$

where $t$ is the thickness of the analyzed sample.

Fig. 13 shows the absorption length for all four refractive aerogel indices calculated from Eq. 团and subtracting the background from the empty light trap measurement. The absorption length increases exponentially for wave lengths ranging from 200 to 400-600 $\mathrm{nm}$ depending on the refractive index. More light is absorbed for the highest refractive index aerogel. For example, at $400 \mathrm{~nm}$ the absorption length for SP-30 aerogel is $20 \mathrm{~cm}$. This value is consistent in magnitude with that reported in Refs. [22, 25]. At about $450 \mathrm{~nm}$, where our PMTs are most sensitive, the absorption length of SP-30 aerogel is $90 \mathrm{~cm}$. The absorption lengths of SP-20, SP-15, and SP-11 aerogels in the range 400-450 
$\mathrm{nm}$ are larger, on the order of a few hundred $\mathrm{cm}$. The measured absorption lengths should also be compared to the total thickness of the aerogel radiator used in the detector, which is $10 \mathrm{~cm}$. This suggests that the effect of absorption in the wavelength range of PMT sensitivity $(380-450 \mathrm{~nm})$ is negligible for all refractive aerogel indices, and even for the worst case, the SP-30 aerogel, the major fraction of the radiated light not be absorved in the aerogel. We note that in the region above 400-600 $\mathrm{nm}$ depending on the refractive index we only report the low limit for the light absorption length.

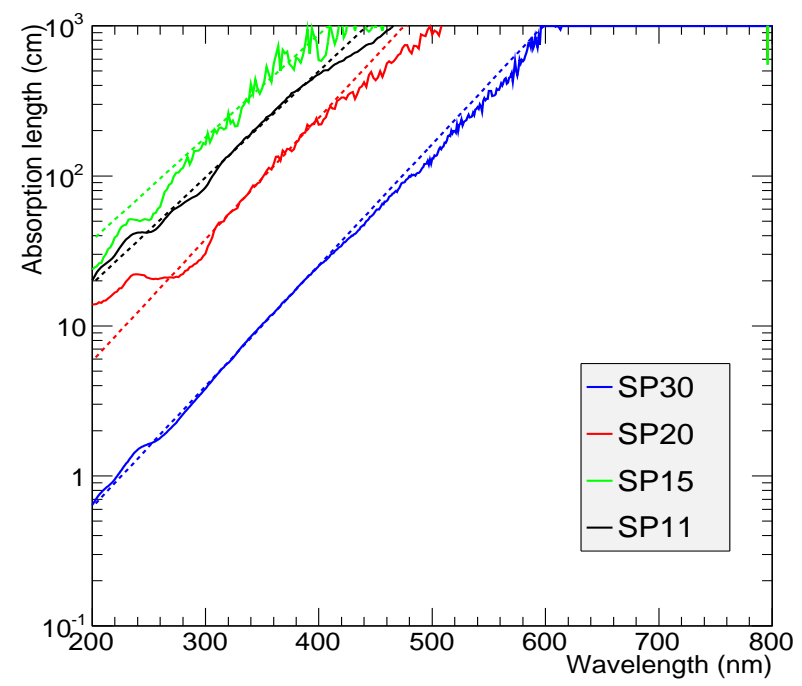

Figure 13: (Color online) The absorption length determined from the forward and backward scattering measurements, e.g., those presented in Fig. 12 for SP-30 aerogel, using Eq. 4

Earlier studies show that for the variety of aerogels the absorption length is nearly constant between 320 and $900 \mathrm{~nm}$, and drops down logarithmically in the range 320-200 nm [22, 25, 30, 31]. Typical values of the absorption length for aerogels with refractive indexes $n=1.010-1.060$ at $\lambda \geq 400 \mathrm{~nm}$ are reported as $>40-60 \mathrm{~cm}$, consistent with the values we measured. 


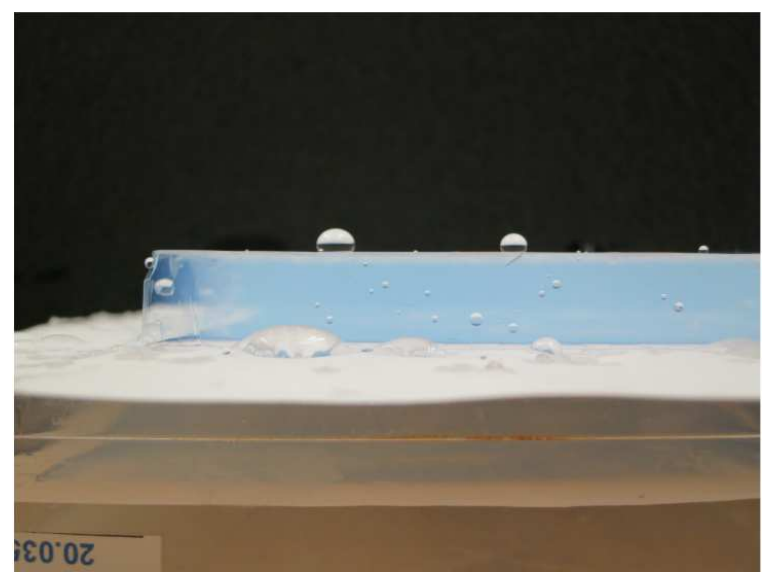

Figure 14: (Color online) Drops of water on the hydrophobic surface of an aerogel tile.

\subsection{Aerogel hydrophobicity}

The density of the aerogel (directly related to its refractive index) can be significantly changed if the aerogel absorbs the humidity from the surrounding environment. To eliminate this effect, some aerogel manufacturers use a hydrophobic coating on the tiles. This makes it possible to use the aerogel material in detectors for a long period of time without requiring special flushing with dry gas or any periodic maintenance.

The aerogel tiles used for the construction of the SHMS Aerogel Cerenkov detector feature such a hydrophilic coating. To check the hydrophobicity of the aerogel, tests were carried out under severe humidity conditions. First, we kept some samples of the tiles for 24 hours at the relative humidity $84 \pm 2 \%$, and later at $91 \pm 2 \%$. No significant change of the aerogel optical properties, e.g., the refractive index, were observed: the average change in refractive index due to humidity was measured to be less than +0.00010 .

Finally, drops of water were added directly on the aerogel surface for a "wettability test" (as shown in Fig. 14), in which the contact angle of the water droplets to the aerogel surface is measured. For a hydrophilic surface the contact angle is expected to be less than 90 degrees, while hydrophobic surfaces have contact angles of more than 90 degrees. Our tests confirm that the aerogel tiles 
used in the SHMS Aerogel Čerenkov Detector are hydrophobic.

\section{PHOTOMULTIPLIER TUBES STUDIES}

The main goal of these studies was to select the best PMTs for the SHMS aerogel detector from a pool of $\sim 70$ PMTs model XP4500B previously used in the BLAST experiment [23, 24] and from $\sim 25$ PMTs model XP4572B previously used in the G0 experiment [32]. All of these are Photonis 10-stage PMTs, with semi-transparent bi-alkali photocathode with peak quantum efficiency $\sim 20 \%$ at wavelength $\lambda \sim 350-450 \mathrm{~nm}$, and gain of $\sim 2 \times 10^{7}$ at an operating high voltage 1800-2100 V. Their internal structure is similar, but the XP4572 photocathode 435 is flat, while the XP4500 photocatode is semi-spherical.

The main requirements for PMT selection were high quantum efficiency, low noise, high gain at relatively low high voltage (HV), and good single photoelectron resolution. For all PMTs the gain and its dependence on high voltage, and relative quantum efficiency were measured. For randomly selected PMTs we also studied photocathode uniformity, and effect of external magnetic fields and shielding on the PMT performance. The final selection of PMTs for the detector consists of 14 PMTs of type XP4572 based on the results of the studies described in the following subsections.

\subsection{Measurements of PMT gain and relative quantum efficiency}

At any operating HV, gain measurements typically begin with localizing the single-electron peak (SEP). The standard method to measure the SEP is to shine a light source on the photocathode and analyzing the resulting spectrum. Here, we used a blue LED with a peak wavelength of $\sim 470 \mathrm{~nm}$. To identify the SEP one needs to gradually reduce the LED driving current until one reaches the state when the position of the spectrum does not depend on light intensity anymore. Further reduction of the light intensity will then only change the proportion between the pedestal (which corresponds to events that do not release a photoelectron from the photocathode) and the SEP. With the SEP position 


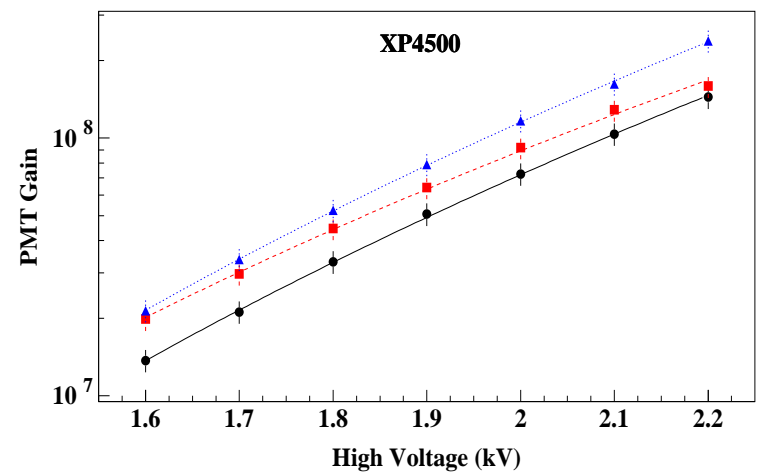

Figure 15: (Color online) Gain versus high voltage for three Photonis XP4500 PMTs. The data are fitted with a function $G \approx \alpha \times x^{\beta \times n}$, where $n=10$ is the number of dynodes, and $\alpha$ and $\beta$ are free parameters. At an operating high voltage of $1.7-1.8 \mathrm{kV}$ the gain of the XP4500 PMT is on average $\sim 3 \times 10^{7}$.

known, the PMT gain at a given HV can be defined from:

$$
\left(N_{S E P}-N_{P E D}\right) \times q_{0}=q_{e} \times G \times k
$$

where $N_{S E P}$ and $N_{P E D}$ denote the mean values of the signal and pedestal amplitudes in ADC channels, $q_{0}$ is the charge per ADC channel 1 , $q_{e} \approx 1.602 \times$ $10^{-19} C$ is the electron charge, $G$ is the PMT gain at given $\mathrm{HV}$, and $k$ is the signal attenuation or amplification factor between the PMT output and ADC input. To eliminate contribution from stray light, a "Dark box" was used for the PMT studies. Fig. 15 illustrates the typical gain dependence on the high voltage for three randomly selected Photonis XP4500 PMTs. The gain, on a logarithmic scale, depends linearly on the applied high voltage and varies from PMT to PMT.

In general, the precision of the gain measurements depends on the accuracy in the positions of the SEP and pedestal, as well as the distance between these two. The uncertainty in these quantities in our measurements is on average

\footnotetext{
${ }^{1}$ e.g., $100 \mathrm{fC} /$ channel for the CAEN V792 QDC
} 
$\sim 10 \%$.

Once the gain of a PMT has been measured the relative quantum efficiency from a signal with a sufficiently large number of photons, e.g., more than a few 100 photons, can be derived from the total charge collected from the PMT anode using

$$
Q=N_{A D C} \times q_{0}=N_{\gamma} \times Q E \times G \times q_{e},
$$

where $Q$ is the total charge, $N_{A D C}=N_{S E P}-N_{P E D}$ is the peak value of the pedestal subtracted signal amplitude in ADC channels, $Q E$ and $N_{\gamma}$ denote the quantum efficiency and number of photons, and the other parameters are as in eq. 5

There are two ways to determine the quantum efficiency (QE) assuming a constant flux of incident light throughout the measurements. In the first method one needs to know the PMT gain and can obtain the relative quantum efficiency from eq. 6.

$$
Q E \sim N_{A D C} / G
$$

In the second method, the relative $Q E$ of a PMT is obtained from the number of detected photoelectrons. At fixed gain and fixed light intensity the number of detected photo-electrons depends only on the PMT quantum efficiency $Q E \propto N_{p e}$. Neglecting contributions from electronic noise and other possible fluctuations $N_{p e}$ can be estimated as inverse square of the normalized width of the detected photoelectron distribution,

$$
N_{p e}=1 / \sigma_{\text {norm }}^{2}
$$

where

$$
\sigma_{n o r m}=\sigma / N_{A D C} .
$$

with $\sigma$ the width of the amplitude distribution determined from a Gaussian fit and $N_{A D C}$ as before the pedestal-subtracted signal amplitude in ADC channels.

For the relative quantum efficiency measurements the LED light intensity was fixed by setting the driving voltage. The PMTs HV were set to a fixed gain of $\approx 2 \times 10^{7}$ (but different high voltage). 
The number of photoelectrons detected can be obtained from both methods. In the first, we first determined the SEP at a minimum LED intensity (at driving voltage $\sim 1 \mathrm{~V}$ ) for each PMT. The PMT amplitude spectrum was then measured at high LED intensity (with a $\sim 4 \mathrm{~V}$ driving voltage, which corresponds to $\sim 6000$ photons). In the second method, the LED intensity was kept fixed at $4.0 \mathrm{~V}$ and the number of photoelectrons was determined from eq. 8 and eq.9. Before starting the relative quantum efficiency measurements, several systematic tests to check linearity of the electronics, LED stability, accuracy of the positioning of the PMTs relative to the LED, and reproducibility of the results were performed. The systematic uncertainty in the measurements due to these factors is about 5\%. For each PMT both methods give a similar (within $15 \%)$ number of detected photoelectrons.

At high LED intensities the XP4500 phototubes produce an average of $\sim 800$ photoelectrons with a standard deviation of $\sim 25$. This corresponds to a quantum efficiency of $Q E \sim 13-14 \%$. For the XP4572 the average number of photoelectrons is $\sim 1100$ with a standard deviation of $\sim 35$, which corresponds to $Q E \sim 18-20 \%$.

Figure [16] shows the results from measurements of a randomly selected sample of ten XP4500 and six XP4572 PMTs with both blue and green LEDs at light intensities of $\sim 1000(\sim 650)$ photons. The XP4572 PMTs detected $\sim 220$ ( 130) photoelectrons, while the XP4500 PMTs detected only $\sim 135(\sim 75)$ suggesting nearly $30-40 \%$ less output signal. Both types of PMTs have similar photocathode spectral sensitivity, at least in the region of blue and green light used in these measurements. The differences in the detected number of photoelectrons thus reflect a real difference in quantum efficiency between these two PMT models. Scans of the PMT photocathode show that the XP4572 effective area is much larger than that for XP4500 (see subsection 4.2), which may explain the observed difference.

The selection criteria for PMTs for the present detector are a combination of the i) highest gain at relatively low high voltage, and ii) the highest relative quantum efficiency. Thus, although the measured relative quantum efficiency 


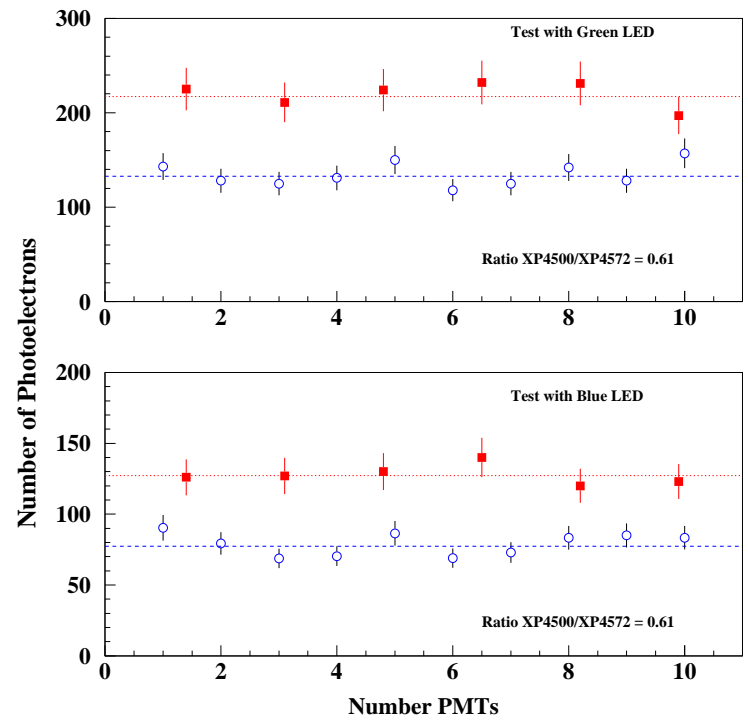

Figure 16: (Color online) Relative quantum efficiencies (number of photoelectrons) for the XP4500 (open circles, blue) and XP4572 type PMTs (solid squares, red). The top (bottom) panel shows data measured with a green (blue) LED, corresponding to intensities of $\sim 1000$ ( 650 ) photons. The XP4500 PMTs have on average $\sim 30-40 \%$ less output signal as compared to the XP4572 PMTs.

for the XP4500 is lower than that of XP4572 PMTs their higher gain could, in principle, still make them suitable for the detector filled with aerogel $n=1.030$. However, for the lower refractive indices the light collection efficiency of the XP4500 is not suitable.

To check for possible improvements of the XP4500 light detection efficiency, we explored the blue wavelength shifting (WLS) paint EJ-298\#2 for the PMT photocathode 2 . A thin $(\sim 100 \mu \mathrm{m})$ and uniform layer of EJ-298\#2 was applied to the phototube window at room temperature. Tests with such WLS for JLab Hall A gas Čerenkov detectors have shown improvements of $\sim 20 \%$ for UV/Blue light [34].

\footnotetext{
${ }^{2}$ The EJ-298\#2 absorbs light with wavelength $(\lambda)$ in the region $300-400 \mathrm{~nm}$ and emits at $\lambda \sim 400-550 \mathrm{~nm}$ (with an efficiency of 70-90\%) 33].
} 
To test the effectiveness of the WLS the signal of one XP4500 PMT was measured with and without WLS. These measurements were performed using the single counter discussed in section 3 with about ten tiles of aerogel of thickness $\sim 10 \mathrm{~cm}$ and nominal refractive index $n=1.030$. No significant difference was observed in the output signal. This result may not be unexpected, since the aerogel material and the reflector on the walls of the single counter absorb more than $90 \%$ of the produced UV light before it can reach the PMT.

\subsection{PMT photocathode uniformity}

Uniformity of the signal across the surface of the photocathode is of concern for large diameter PMTs. A setup that can repeatedly reposition a directed light source, e.g., an LED, to different positions in front of the PMT was thus constructed to test the detection efficiency over the photocathode surface.

The apparatus used for these measurements is shown in Fig. 17, A two-axis stepper motor setup (Velmex) was used which automatically repositions a blue LED attached to a 0.029-inch collimator and an optical fiber to direct the light to specific points on the PMT photocathode.

An adjustable jack was used to position the PMT in the center of the rail's ${ }_{525}$ limits and the entire setup was installed inside a custom dark box. The motor controller was programmed to scan in 100 by 100 steps. At each point, the motor controller output triggers the DAQ system and a controllable pulse to the LED, collecting 30 data points per location. At each flash of the LED, an analog to digital converter integrates the current received by the PMT with a conversion factor of $0.25 \mathrm{pC}$. The stepper motor moved 450 steps at a step length of $0.0025 \mathrm{~mm}$ with every motor turn, resulting in a scan resolution of 1.2 $\mathrm{mm}$. The total distance that each axis covered was $112.5 \mathrm{~mm}$ so that an effective area of about $120 \mathrm{~mm} \times 120 \mathrm{~mm}$ of the PMT was scanned. The collected data were converted from the DAQ intrinsic CODA format to a plain ASCII format ${ }_{535}$ for further analysis. 


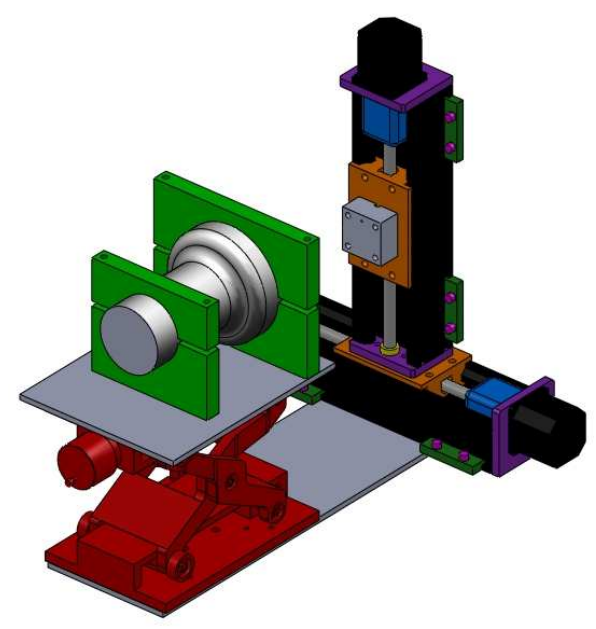

Figure 17: (Color online) Schematic of the PMT photocathode scanning system. The Velmex stepper motor setup allows for moving a blue LED along the two axes covering the full area of the photocathode.

Fig 18 shows a typical two-dimensional effective photocathode area of the XP4500 (top panel, a) and XP4572 PMTs (bottom panel, b). For the XP4572 PMT, the region of highest efficiency covers almost the entire scanned region. In contrast, the region of highest efficiency for the XP4500 only covers about half the scanned area. The maximum difference of the signals mean values across the photocathode is about $30 \%$. This may explain our observation of low quantum efficiency for the XP4500 PMTs discussed in subsection 4.1.

In general, such non-uniformities of the photocathode response are not important for diffusive light collection as photons originating from the aerogel radiator reach the PMT surface only after several reflections and nearly uniformly illuminate the photocathode. Nevertheless, the non-uniformities need to be taken into account in the analysis, since it affects the PMT average quantum efficiency, and so the overall number of detected photoelectrons. 


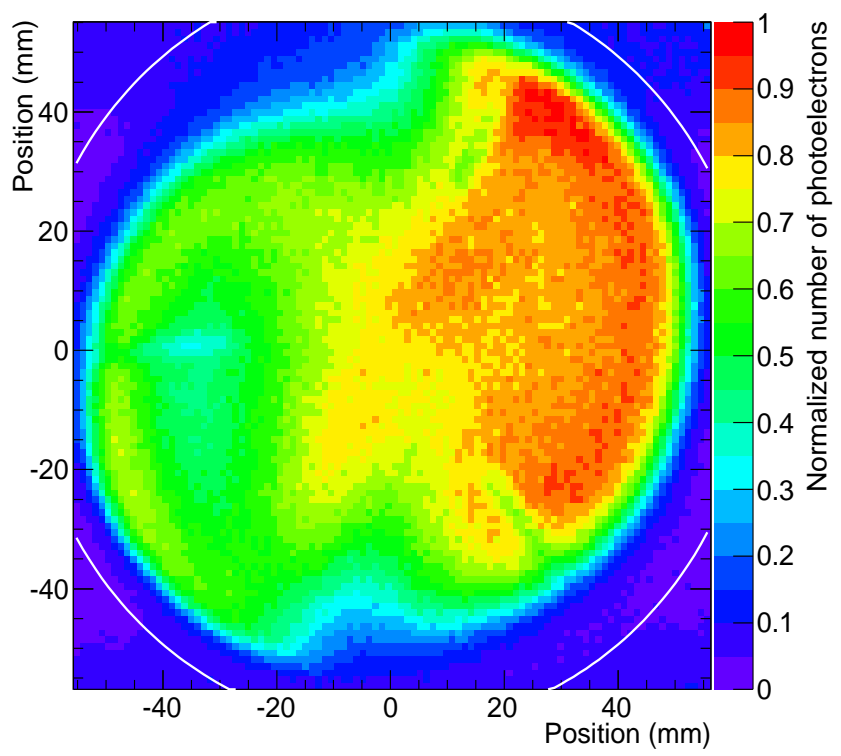

(a) PMT XP4500

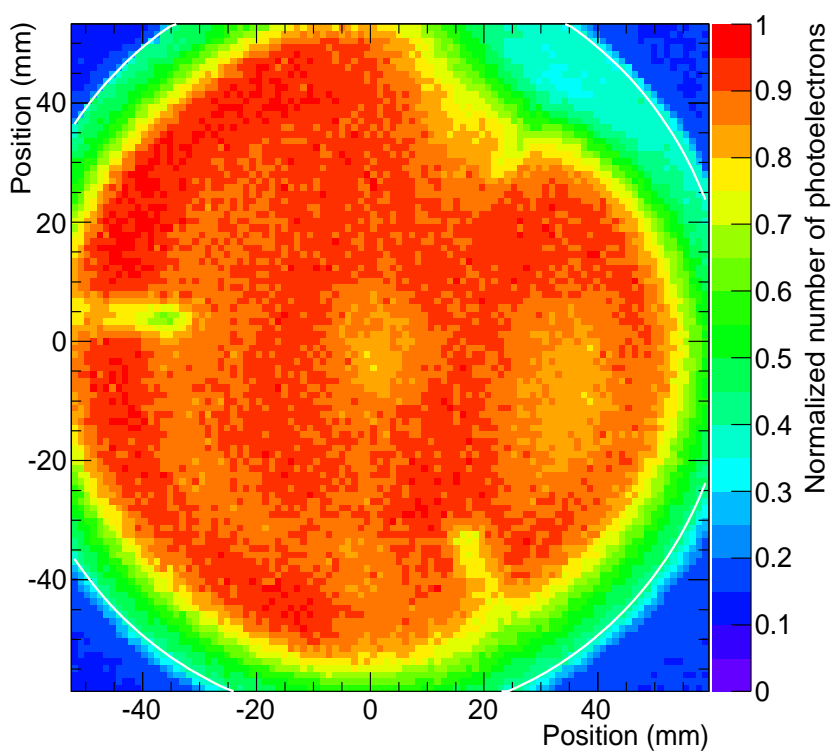

(b) PMT XP4572

Figure 18: (Color online) Two-dimensional scans of the number of detected photoelectrons (normalized) over the areas of the XP4500 (top. ${ }_{34}$ ) and XP4572 (bottom, b) PMT photocathodes. 


\subsection{Magnetic shielding}

Photomultiplier tubes do not operate properly when exposed to magnetic fields. Magnetic fields may deflect electrons from their normal trajectory and cause a loss of photoelectron collection efficiency and gain. The impact on the performance depends both on the PMT type, e.g., their diameter and internal structure, and on the PMT orientation in the field. Covering the PMT with a cylindrical shield case longer than its length by at least half the shield case inner diameter is thus common practice. The performance of this type of shielding in small magnetic fields, like the Earth's field, and a supplementary method to optimize the PMT performance in larger magnetic fields is discussed below.

\subsubsection{Performance in weak external magnetic fields}

For relatively small fields like the Earth field $\sim 0.3-0.6 \mathrm{G}(30-60 \mu \mathrm{T}) \mu$ metal typically provides sufficient shielding. To study the effect of the Earth's magnetic field on the 5-inch PMT Photonis PMTs used in the SHMS aerogel Čerenkov detector the PMT axis was oriented along the direction of the Earth magnetic field (axial). An LED-fiber coupled light source was used to illuminate the photocathode. Data were taken both with the blue LED directly fixed on the photocathode, and with the PMT at a distance of $10 \mathrm{~cm}$ from the light source to cover a wide surface of the photocathode as uniformly as possible. The LED intensity was kept constant during the measurement.

In the first measurement the PMT without magnetic shield was rotated around its axis (which is perpendicular to the photocathode) in $30^{\circ}$ steps clockwise to establish a baseline of the azimuthal dependence of its amplitude on the external field. The result is shown in Fig. 19. The PMT amplitude depends strongly on the azimuthal angle varying in a $\cos (2 \phi)$ pattern independent of the position of the light source, be it directly fixed on the photocathode or at 575 a distance of $10 \mathrm{~cm}$ away from it. Here, zero degree corresponds to the PMT pointing North. The azimuthal variation corresponds to an amplitude roughly $40 \%$ of the signal.

For the subsequent measurements, the PMT was enclosed in a cylindrical 


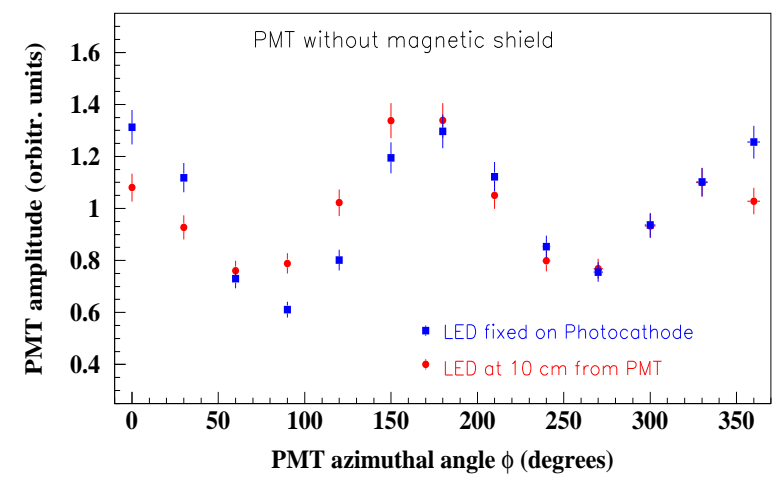

Figure 19: (Color online) The PMT amplitude as a function of its orientation (measured in terms of the azimuthal angle $\phi$ ) in the Earth's magnetic field without magnetic shielding. Zero degrees is defined as the PMT axis oriented along the Earth magnetic field.

magnetic $\mu$-metal shield of inner diameter $\sim 133 \mathrm{~mm}(5.236 ")$ and thickness $\sim 1$ mm (0.040"). In these measurements, the degree to which the PMT could still be affected by the external field was tested by shifting the photocathode relative to the edge of the magnetic shielding cylinder. A distance of "zero" means that the PMT photocathode is positioned right at the edge of the magnetic shielding cylinder. The horizontal axis is perpendicular to the PMT photocathode surface and measurements were performed for two orientations of the PMT with respect to the Earth's field, at azimuthal angles of 180 and 60 degrees, respectively. Here, 180 degrees corresponds to an orientation parallel, but in opposite direction to the Earth's magnetic field, while 60 degrees is a large angle near perpendicular. The performance of the magnetic shield for all cases is illustrated in Fig. 20.

For the photocathode located outside of the magnetic shielding up to distances of $\sim 5 \mathrm{~cm}$, the Earth's field does not have any notable impact on the PMT. For the photocathode located fully within the magnetic shield cylinder (zero or positive distances), or even moving the magnetic shield cylinder a distance of $5 \mathrm{~cm}$ behind the photocathode, the weak magnetic field has no effect, for either angular orientation, and the magnetic shielding remains effective. For 


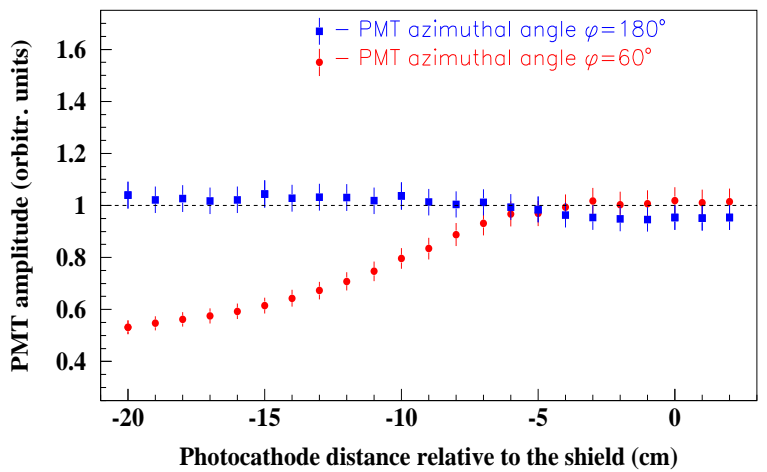

Figure 20: (Color online) The PMT amplitude as a function of photocathode distance relative to the shield for its two orientation (azimuthal angle $\phi=60$ and 180 degrees) relative to Earth's magnetic field.

an orientation of $\phi=180$ degrees the magnetic shielding remains even up to a distance of the magnetic shield cylinder $20 \mathrm{~cm}$ behind the photocathode, there is no effect independent of the photocathode's position inside the shielding cylinder. On the other hand, moving the photocathode more than $5 \mathrm{~cm}$ outside of the magnetic shielding for an azimuthal angle of $\phi=60$ degrees has a large effect: the effect of the Earth's magnetic field can again reduce the amplitude, by up to $40 \%$, to closely mimic the unshielded situation.

These results have an important design impact for our detector, the magnetic shield of the PMTs only need to be extended up to edge of the photocathode. Based on the results of the studies the choice of a $\sim 1 \mathrm{~mm}(0.040 ")$ thickness $\mu$-metal cylinder with inner diameter of $\sim 133 \mathrm{~mm}(5.236 ")$ is confirmed, in order to shield the PMTs of the present detector from weak external magnetic fields ( $<1$ Gauss). The PMT magnetic shielding is aligned with the edge of the photocathode.

\subsubsection{Performance in stronger external magnetic fields}

In the SHMS detector enclosure where the aerogel Cerenkov detector will be located fringe fields from the spectrometer magnets are expected to be less than 
0.5 Gauss. However, the design of the present detector is flexible and includes 615 a method to allow PMT operation in fields up to 5 Gauss. This would allow operation of the detector at a location in the detector stack that is close to the SHMS dipole, e.g., before the first hodoscope plane to further reduce knock-on or $\delta$-electrons, should it be needed for future experiments.

An effective technique to minimize the effect of spectrometer residual mag${ }_{620}$ netic fields is the use of bucking coil systems [35]. The current sent through the bucking coil produces a magnetic field opposite in direction to the external magnetic field from the spectrometer. By regulating the coil current one can thus reduce (compensate) the effect of external fields on the PMTs.

The experimental setup used for the bucking coil studies is shown in Fig. 21 ${ }_{625}$ The PMT and its magnetic shield were placed inside an aluminum tube of the same type as those that are used to hold the PMTs in place in the aerogel Čerenkov detector itself. The external magnetic field simulating the spectrometer residual fields was provided by a Helmholtz coil producing a magnetic field of about 5 Gauss. In this setup the magnetic field is in linear relationship with ${ }_{630}$ the current through the Helmholtz coil., which allows for calculating the field for a given current directly. 


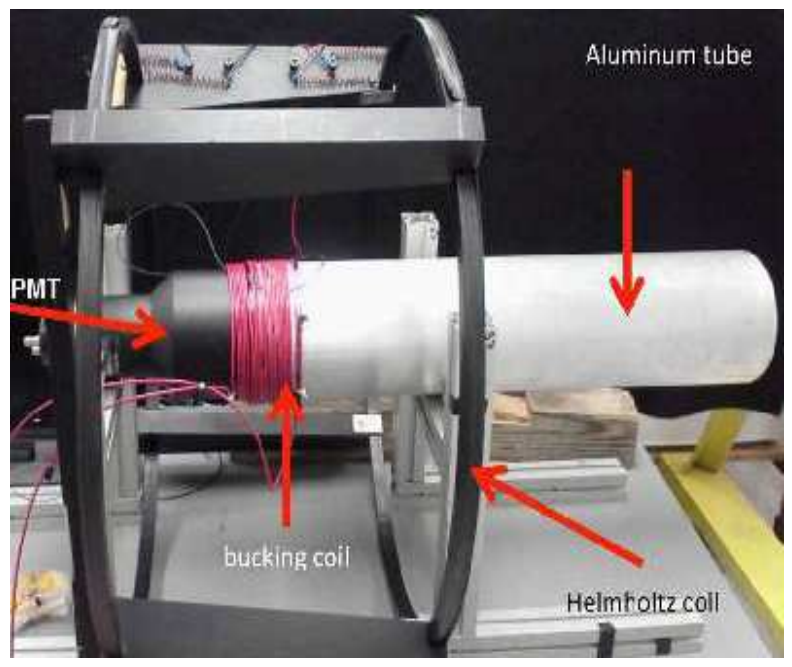

Figure 21: (Color online) Experimental setup developed for bucking coils studies.

Without external magnetic field the PMT amplitude depends logarithmically on the operating high voltage. When the magnetic field was applied, the PMT gain (driven by the secondary emission of the dynodes) was not significantly affected for field strengths ranging between 1-10 Gauss. The photoelectron collection efficiency (from photocathode to first dynode), however, decreases significantly even for relatively small fields of 1-2 Gauss resulting in a 20-50\% loss of signal. This confirms that the decrease in PMT efficiency is due to photoelectrons acted on by the magnetic field before they arrive at the dynode 640 cascade.

The PMT signal recovery using a bucking coil system is illustrated in Fig. 22 For the signal to be fully recovered, the net magnetic field inside the PMT needs to be zero. This happens in our setup roughly when a current of $4.6 \mathrm{~A}$ is applied to the 20-turn bucking coil, allowing for an almost $100 \%$ signal recovery. Up to ${ }_{645}$ an applied current to the bucking coil of about $2 \mathrm{~A}$ the net magnetic field is large due to the external $5 \mathrm{G}$ magnetic field applied, and most of the signal is lost. As the bucking coil current increases beyond $2 \mathrm{~A}$, the magnetic field it produces eliminates part of the external field, until their magnitudes are matched and 
they cancel each other achieving a net field of zero at the $\sim 4.6$ A current. Once field, at bucking coil current above $4.6 \mathrm{~A}$, the signal recovery starts to decrease again as the net field inside the PMT becomes larger. For the present detector the setup thus includes a flexible design allowing to add the bucking coil feature, to fully compensate for external fields of about 5-10 G.

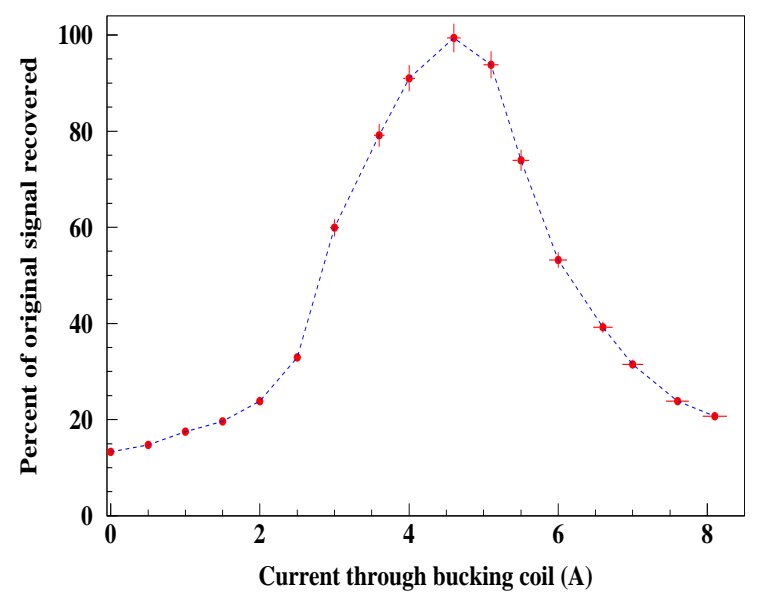

Figure 22: (Color online) Recovery of the PMT signal in an external (few-Gauss) field by applying current to a bucking coil. When the external and bucking coil fields cancel, the signal is fully recovered.

\section{Detector Performance}

The efficiency and background rejection capability of the aerogel Čerenkov detector depends on the geometry optimization, on the thickness of the radiator, and the location in the SHMS detector stack. Extensive Monte Carlo studies and tests with a prototype of the SHMS aerogel Cerenkov detector were performed to optimize the aerogel thickness for the best kaon detection efficiency and highest rejection factor for protons. Details of the Monte Carlo (MC) program and the material parameters used can be found in section 8.1. For quality assurance and to verify light collection and uniformity, the full detector was 
tested with cosmic rays before and during the filling of the aerogel trays, and again after the installation in the SHMS detector hut. This section describes the detector performance including detection efficiency and background rejection, sub-threshold background rejection, as well as light collection and uniformity.

\subsection{Projected kaon detection efficiency and proton suppression}

The ultimate performance of the detector for kaon detection efficiency is correlated with its performance for proton suppression. One can reach higher kaon detection efficiency at the cost of less proton suppression. This choice will be based on the actual kaon to proton ratios as present in the experimental configuration. Some examples are presented in Fig. 23, which illustrates the projected kaon detection efficiency and proton suppression for the SHMS Aerogel Čerenkov Detector as a function of particle momentum for two different cuts on the number of photoelectrons. The four panels represent projections for the different refractive indices, $n=1.030,1.020,1.015$ and 1.011, respectively.

For the regions of interest defined by the threshold momenta listed in Table I, the kaon detection efficiency for a cut $N_{p e}>1$ (open squares) is no less than $90 \%$ for all refractive indices except for the lowest refractive index, where it is about $85 \%$. The proton suppression (open squares) is on the order of 50-70:1 in the respective region of interest for all refractive indices except for the SP-11. There, the projected proton suppression is about 100:1 for momenta above 5.4 $\mathrm{GeV} / c$ The projected detection efficiency and proton suppression are suitable for the experiments described in section 1 .

The kaon signal detection efficiency and proton rejection factor can be further optimized by requiring different (stricter) cuts on the number of photoelectrons. As an example the open (solid) circles represent the projections for cuts on $N_{p e}>5$ (SP-30), $N_{p e}>4$ (SP-20), $N_{p e}>3$ (SP-15), and $N_{p e}>2$ (SP-11).

${ }_{690}$ For these cuts, the proton rejection factor increases to about 150:1. However, this comes at the expense of a decreased kaon signal detection efficiency, which would be reduced to $70 \%$ on average in the region of interest. 

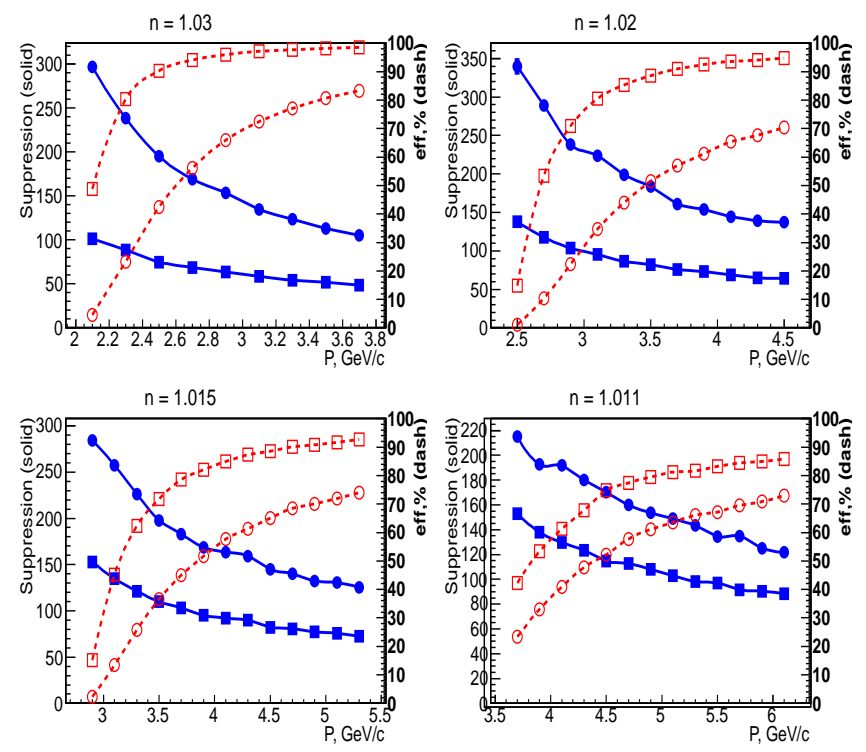

Figure 23: (Color online) The projected kaon detection efficiency (open symbols) and proton suppression (solid symbols) versus particle momentum for the SHMS Aerogel Čerenkov Detector with nominal refractive indices of $n=1.030,1,020,1.015$ and 1.011. The open (solid) squares represent projections for a cut on the number of photoelectrons of $N_{p e}>1$. The open (solid) circles represent the projections for cuts $N_{p e}>5$ (SP-30), $N_{p e}>4$ (SP-20), $N_{p e}>3$ (SP-15), and $N_{p e}>2(\mathrm{SP}-11)$.

\subsection{Sub-threshold background rejection}

The main limitation for the background rejection capability of threshold detectors is the production of knock-on electrons (also called $\delta$-electrons). The general impact of $\delta$-electrons on aerogel detector rejection capabilities is discussed in Refs. [36, 37]. There, the impact of $\delta$-electrons on a measurement was estimated to be a few percent depending on geometry, details of the construction and location of the detectors. In general, the probability to produce $\delta$-electrons by particles with a momentum below the Cerenkov radiation threshold was shown to increase with radiator thickness.

The SHMS Aerogel Čerenkov Detector is located after the noble-gas Čerenkov, the pair of drift chambers, the first pair of scintillation hodoscope planes (1X 
and $1 Y$ ) and the heavy gas Čerenkov detector. A non-negligible amount of $\delta$ electrons will be produced in the materials of these detectors and in the air-gaps between them, and some of these may have enough energy to penetrate inside the effective volume of the aerogel detector and produce themselves Cerenkov light. Thus, protons with a momentum below the threshold momentum for generating Čerenkov light in aerogel may produce $\delta$-electrons in the detectors located upstream of the aerogel Čerenkov counter or/and in first several layers of the aerogel itself. Since a diffusion-type aerogel detector only provides information about the number of detected photons, there is no way to separate these events from the real kaon events. These $\delta$-electrons may reduce the rejection capability of the aerogel detector and contribute to fluctuations in the signal (see for example Ref. [38]), and thus merit a more detailed discussion.

We have estimated the number of $\delta$-electrons which can be generated in the SHMS Aerogel Čerenkov Detector. For simplicity, first we took into account the aerogel detector alone. For a conservative estimate we assumed an entrance window thickness of $1.5 \mathrm{~mm}$ (about 30\% thicker than the actual thickness of the detector entrance window).

In general, the spectrum of $\delta$ electrons that have sufficiently high energy to produce Čerenkov light is given by [39]

$$
N(E) d E=0.30058 \frac{m c^{2}}{\beta^{2}} \frac{Z}{A}\left(1-\frac{\beta^{2} E}{E_{m}}\right) \frac{1}{E^{2}} d E,
$$

where $N(E)$ is the number of $\delta$-electrons of kinetic energy $E$ produced per $\mathrm{g} / \mathrm{cm}^{2}$ of a target of given $(Z / A), \beta$ is the proton velocity, $m$ is the electron mass, and $E_{m}$ is the maximum kinetic energy transferred to the $\delta$-electron in an ion-electron collision.

The $\delta$-electron spectrum is falling rapidly at electron energies near the Čerenkov threshold. The total number of $\delta$-electrons capable of producing Čerenkov light increases slowly with the primary energy. However, the total energy contained in them rises rapidly as does the average energy [40]. The total light yield generated by these $\delta$-electrons can be calculated, in principle, taking into account the light yield of stopped electrons, which is for most materials near minimum 
ionizing until it is below threshold. However, in practice this becomes complicated. Significant contributions from $\delta$-electrons to the signal can originate from electrons with energies well beyond threshold, of $20 \mathrm{MeV}$ or more [40]. To include all cascading effects in all materials for such higher-energy electrons would require a solution to the transport equation, which is beyond the scope of this article.

Instead, we make the approximation that below some cut-off energy the $\delta$-electrons contribute according to equation 10 while above this energy the contribution equals that of an electron with the cut-off energy. We require the $\delta$-electrons produced to be within the angular acceptance defined by both the distance to the detector and the aerogel detector effective area, and applied a $E>2 \mathrm{MeV}$ cut of energy. The threshold momentum for electrons in aerogel of nominal refractive index $n=1.030$ is $1.62 \mathrm{MeV}$, so with a $2 \mathrm{MeV}$ energy cut-off we require this to be slightly above the threshold. The results are shown in Fig. 24.

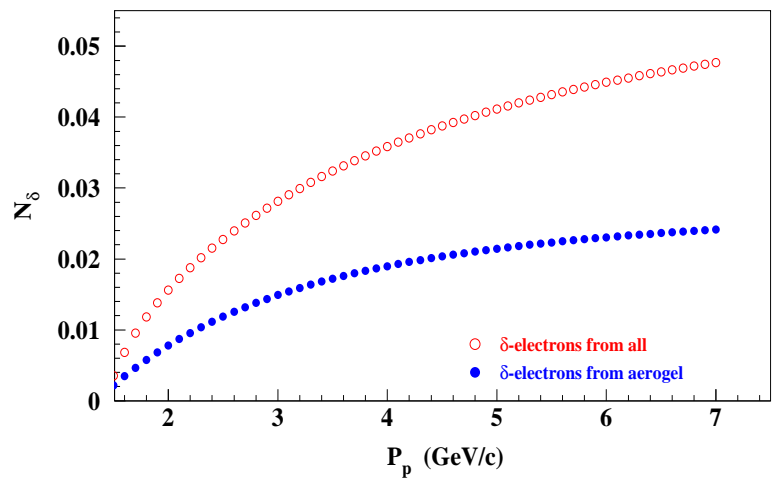

Figure 24: (Color online) Probability to produce $\delta$-electrons versus proton momentum. The solid (blue) circles are for a standalone aerogel detector. The open (red) circles take into account all materials of windows and detectors in the shielded SHMS detector hut before the location of the aerogel detector.

In general, the number of $\delta$ electrons increases with material thickness. In Fig. 24 we show both the probability for protons in the $2-7 \mathrm{GeV} / c$ momentum 
range to produce $\delta$-electrons in the aerogel detector as a standalone detector (closed circles) and taking into account all materials in the shielded SHMS

750 grows with increasing momentum as expected.

The probabilities shown are most likely an overestimate. To estimate the actual effect of $\delta$-electrons on the experiments one has to also take into account the particle identification properties of the other detectors available in Cerenkov detector performance. This is particularly important for the light collection efficiency of the detector's lowest nominal refractive index (SP-11), where the expected signal is small, as shown from the calculations in 3.3

One possible optimization is that of the reflector material in the diffusion 775 used reflector material, however its reflectivity is around $96 \%$ in the wavelength range of the PMTs $(\lambda \sim 350-450 \mathrm{~nm})$ and one expects several light bounces 
in the detector. We studied the reflectivity of several materials often used for detector construction, such as teflon, aluminized mylar, and GORE reflector, aiming to improve the light collection for the lowest index trays and the diffusion box. Measurements of reflectivity of these materials were done using the reflectance port of the integrating sphere shown in Fig. 11, Figure 25 compares the measured reflectivity of GORE $(1 \mathrm{~mm})$ and Millipore, highlighting the spectral region of high efficiency of the PMTs. The $1 \mathrm{~mm}$ thick Gore diffusive reflector material (available in sheet sizes $12^{\prime \prime} \times 12^{\prime \prime}$ ) has a reflectivity greater than $99.7 \%$. The also available $3.2 \mathrm{~mm}$ thick reflector material provides an even higher reflectance [19].

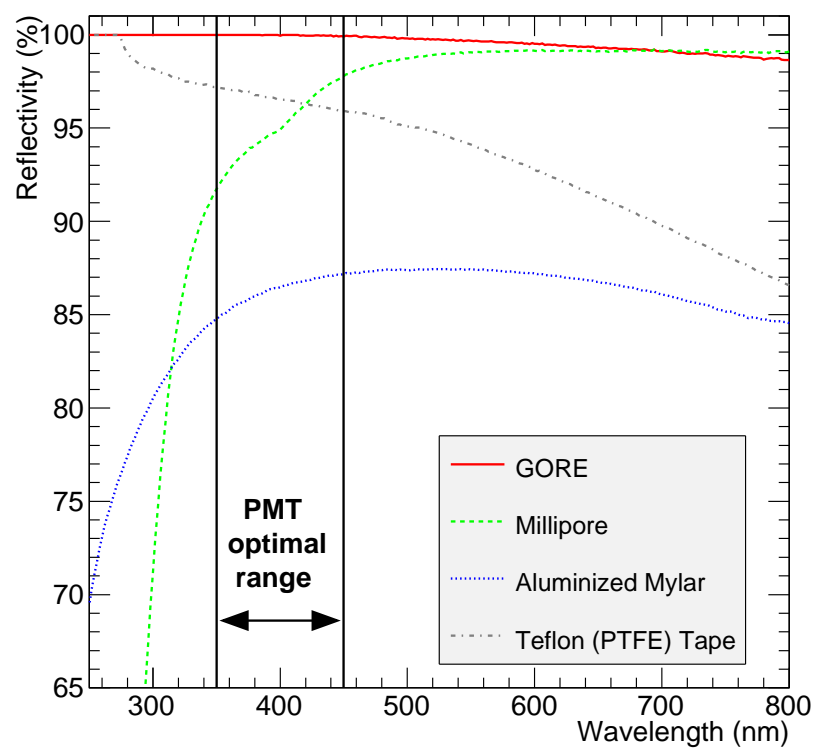

Figure 25: (Color online) Reflectivity of several materials commonly used for covering the interior of detectors. Gore diffusive reflector material has the highest reflectivity where PMTs are most sensitive $(\lambda \sim 350-450 \mathrm{~nm})$ followed by Millipore. In the SHMS Aerogel detector, we used Millipore to cover the trays with aerogel refractive index $n=1.030$ and $n=1.020$, and GORE reflector for the trays with $n=1.015$ and $n=1.011$ to optimize the light collection.

Fig. 26] shows a Monte Carlo simulation of the detector performance if one 
covers the detector with Gore reflector of different thicknesses, as a ratio to the

base detector design with millipore. In some cases only sections of the detector was covered with the Gore reflective material to illustrate that the sensitivity to the higher reflective material is not uniform over the inner detector area. The simulation studies suggest that lining the sides of the diffusion box where the PMTs are housed with the higher reflective material does not make much difference, and that much of the improvement in light collection efficiency is already reached by lining the detector with Gore reflective material of $1 \mathrm{~mm}$ thickness. Covering the surface of the backplane of the detector's diffusion box with $0.5 \mathrm{~mm}$ thick Gore reflective material already gives a little over $20 \%$ improvement in light collection efficiency as compared to the base design with Millipore only. The ultimate performance is reached by covering the entire diffusion box with Gore reflective material of $3.2 \mathrm{~mm}$ thickness, rendering a little over $40 \%$ improvement in light collection efficiency as compared to the base design. We chose an intermediate solution and lined $60 \%$ of the diffusion box with $3.2 \mathrm{~mm}$ and $40 \%$ with $1 \mathrm{~mm}$ thick Gore reflective material.

The average number of detected photoelectrons after (before) replacing Millipore with Gore diffusive reflector in the entire diffusion box is $\sim 12(\sim 9)$ for the aerogel with refractive index $n=1.030(\mathrm{SP}-30)$ and and $\sim 8.0(\sim 6)$ for $n=1.020$ (SP-20). The use of the XP4572 PMTs instead of XP4500 in the diffusion box in turn improved the performance of the detector by $\sim 35-40 \%$ (see section 4.1).

${ }_{810}$ Thus, Gore reflective material and XP4572 phototube have generally resulted in improving the characteristics of the detector by $\sim 70 \%$. This improvement is most significant for the two lower refractive aerogel index trays, where the expected number of photoelectons is small. 


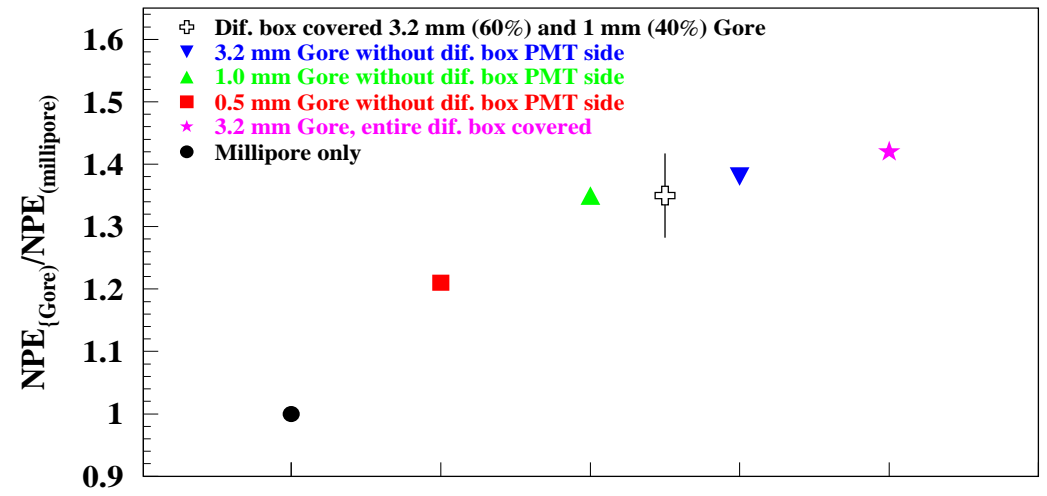

Figure 26: (Color online) Ratio of number of photoelectrons as predicted by simulation for lining (sections of) the detector with different thickness of Gore reflective material relative to lining the detector with Millipore. The open cross shows an experimental measurement for the final chosen configuration with the detector covered for 60 (40)\% with $3.2(1.0) \mathrm{mm}$ thick Gore reflective material, in good agreement with the simulated ratios.

\subsubsection{Measurements with beam}

For a confirmation of the light collection efficiency, we tested the aerogel with nominal refractive index of $n=1.030$, one of the aerogel materials previously used in experiments at MIT-Bates, during the P-349 experiment at CERN, whose first run was completed successfully in December 2014. The particles of interest in this experiment are antiprotons of momentum $3.5 \mathrm{GeV} / c$ produced by the $24 \mathrm{GeV} / c$ momentum proton beam of the CERN/PS along with other secondary particles of the same momentum. These antiprotons have a velocity of $\beta=0.966$. Pions of the same momentum have a velocity close to the speed of light, $\beta=0.9992$. The aerogel thus allows for discriminating these hadrons. Initial analysis suggests the aerogel to indeed perform as expected lending further confidence for the foreseen Aerogel Čerenkov detector's performance in the SHMS. 


\subsubsection{Measurements with Cosmic Rays}

For an initial check, both during the assembly of the various aerogel trays, layer by layer, and after final assembly before installation, the SHMS Aerogel Cerenkov Detector was tested with cosmic rays. For these tests, the detector was positioned horizontally, with the diffusion box on top of the aerogel tray. Two trigger scintillator counters aligned with respect to each other were positioned above and below the detector.

The signal pulses from the scintillators were discriminated and fed into a

835 Logic Unit to form a coincidence trigger providing a "Gate" for a CAEN V792 charge integrating QDC module, or alternatively as a "Start" for a TDC module. The QDC sensitivity was set to $100 \mathrm{fC} /$ channel. The signals generated by cosmic ray muons from all 14 PMTs (7 from each side of the diffusion box) were sent to a passive splitter (50:50). Then, one output was sent to the QDC module through a $\sim 150$ ns delay line to fit within the gate generated by the trigger scintillators. The second output was sent to a discriminator module (Phillips 708) and then to a TDC module. The latter can be used for timing measurements, but these were not the main goal of the current tests. All information from the QDC were read out by a CODA data acquisition system. The offline analysis was done using the ROOT analysis package.

We have checked the stability of the results - the total sum of photoelectrons, with respect to (i) the ADC gate width by changing it from 150 ns to $200 \mathrm{~ns}$; (ii) the timing of the individual PMT signals relative to the gate by shifting signals by $\pm 50 \mathrm{~ns}$; and (iii) the gain of PMTs by changing high voltages by $\pm 100 \mathrm{~V}$. No significant variation in the total sum of photoelectrons measured was found.

The effect of the low-energy components of cosmic rays on the data were first checked, by comparing measurements with and without a $25 \mathrm{~cm}$ thick lead filter installed above the lower trigger scintillator. Our measurements show that the total signal amplitude did not change significantly after filtering out these low-energy components (see Fig. 27). 


\section{SP20, Summed signal, normalized}

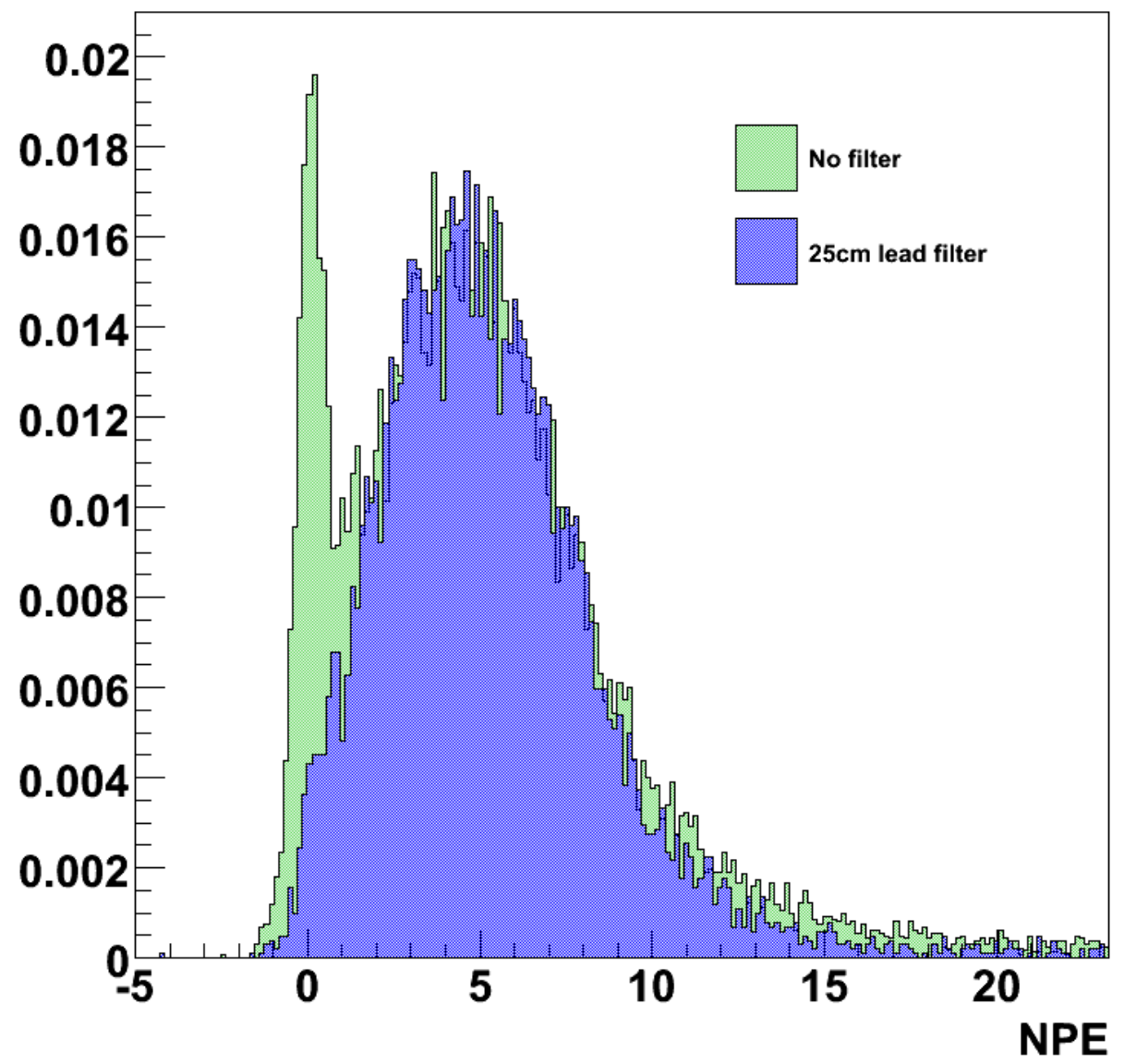

Figure 27: (Color online) Signal distributions from cosmic rays passing through the center of the SHMS aerogel detector filled with 7 layers $(7.7 \mathrm{~cm})$ of SP-20 type aerogel, both with (blue) and without (green) a $25 \mathrm{~cm}$ lead stack acting as low-energy filter placed between the trigger scintillators.

The level of background was determined using the cosmic rays passing through empty space - an area where there were no aerogel tiles stacked - in the detector. Along with pedestal events, the single photoelectron signal was detected, albeit with low intensity, in all the detector PMTs indicating scintillation in air and reflector materials within the detector. These signals became more prominent 
when Čerenkov light from the cosmic rays passed through a $10 \mathrm{~cm}$ thick aerogel radiator of refractive index $n=1.030$. Fig. 28 shows a comparison of the two signals.

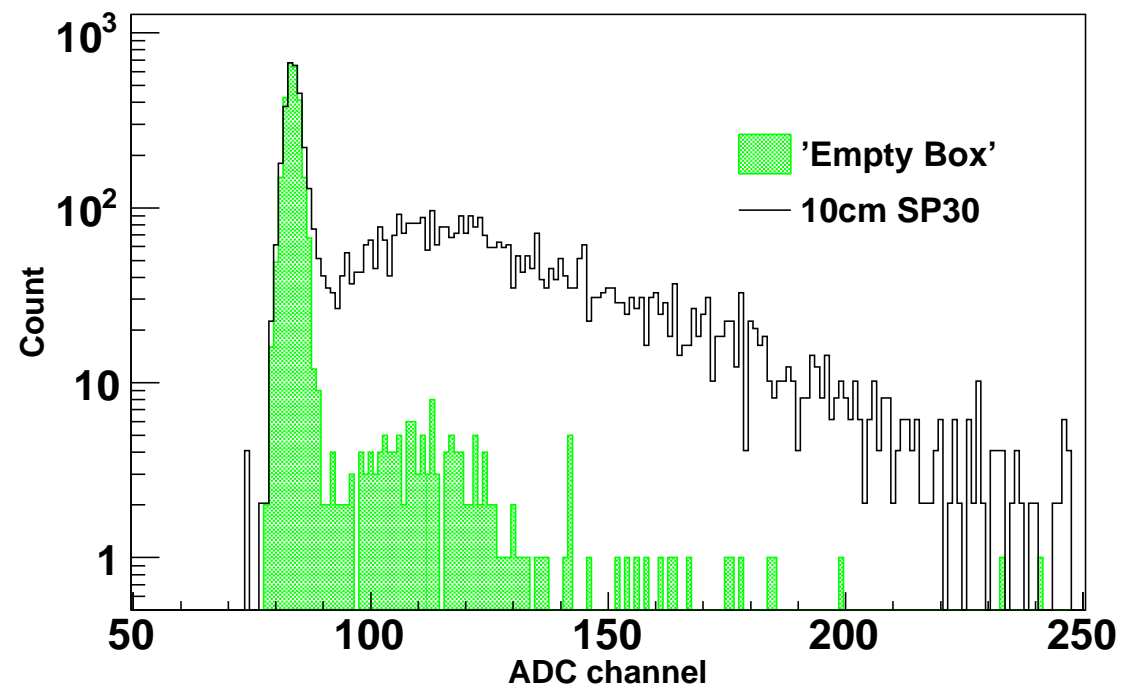

Figure 28: (Color online) Representative ADC spectra of a detector PMT, triggered by cosmic rays passing through an area where a $10 \mathrm{~cm}$ thickness of SP-30, $n=1.030$, aerogel material was stacked and an area where no aerogel radiator material was stacked ('Empty Box'). The histograms are normalized to a common maximum height.

Fig. 29 illustrates a double Gaussian fit to a typical ADC spectrum of the detector PMT signals, to identify both the pedestal and the single photoelectron (SPE) peak positions. The total (summed over all channels) detector signal was obtained from the pedestal subtracted SPE position. This then allows us, after summing all detector PMTs, to convert the measured ADC spectra to the anticipated number of photoelectrons used in further figures. 


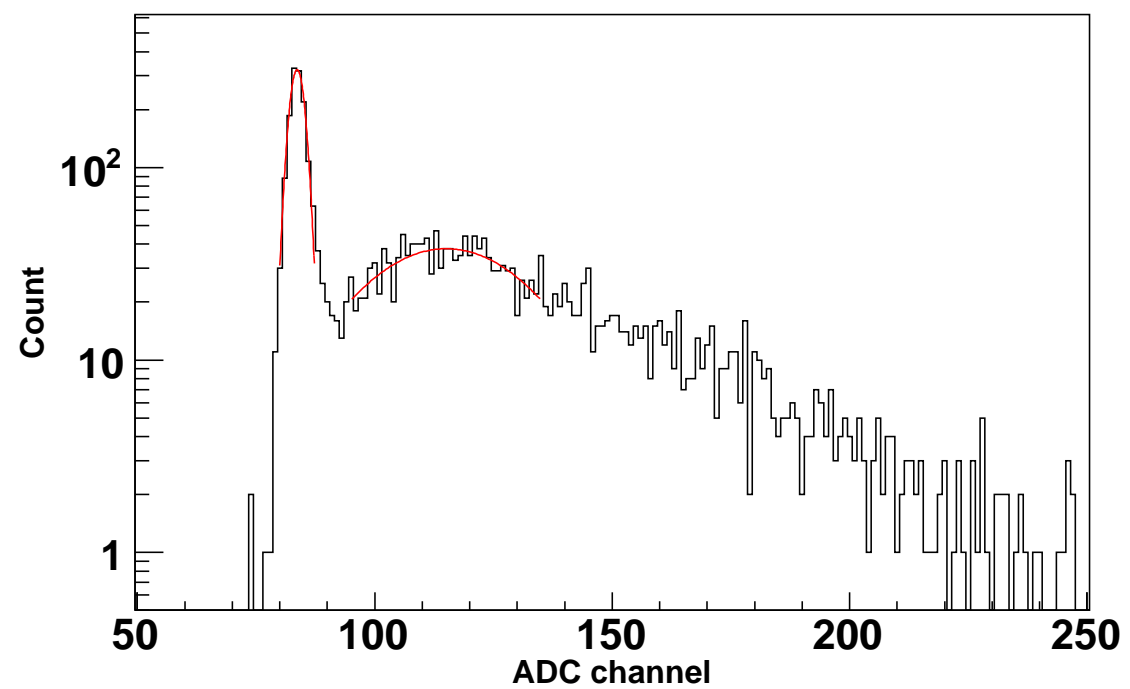

Figure 29: (Color online) Typical example of a Gaussian fit to the ADC spectrum of one of the detector PMTs. The mean values of these Gaussian fits allow one to identify the pedestal and single photoelectron peak positions.

We first placed a single stack of aerogel tiles in the center of the aerogel box. Thus, the stack's area was that of a single tile, i.e. $11 \times 11 \mathrm{~cm}^{2}$. The light collection-performance of the detector in this configuration was tested by taking data with aerogel tiles stacked up to $10 \mathrm{~cm}$ high. Fig. 30, shows the results, converted to the number of photoelectrons, for two nominal refractive indices, $n=1.015$ and 1.030, in comparison to the case with no aerogel material present. The signals for the two refractive indices differ by a factor of $\sim 2$, as expected from analytical calculations. Cosmic rays passing through an area where no aerogel is stacked ('empty box') produce a, slightly asymmetric, peak at zero with a width of $\sim 1$ p.e. (HWHM). 


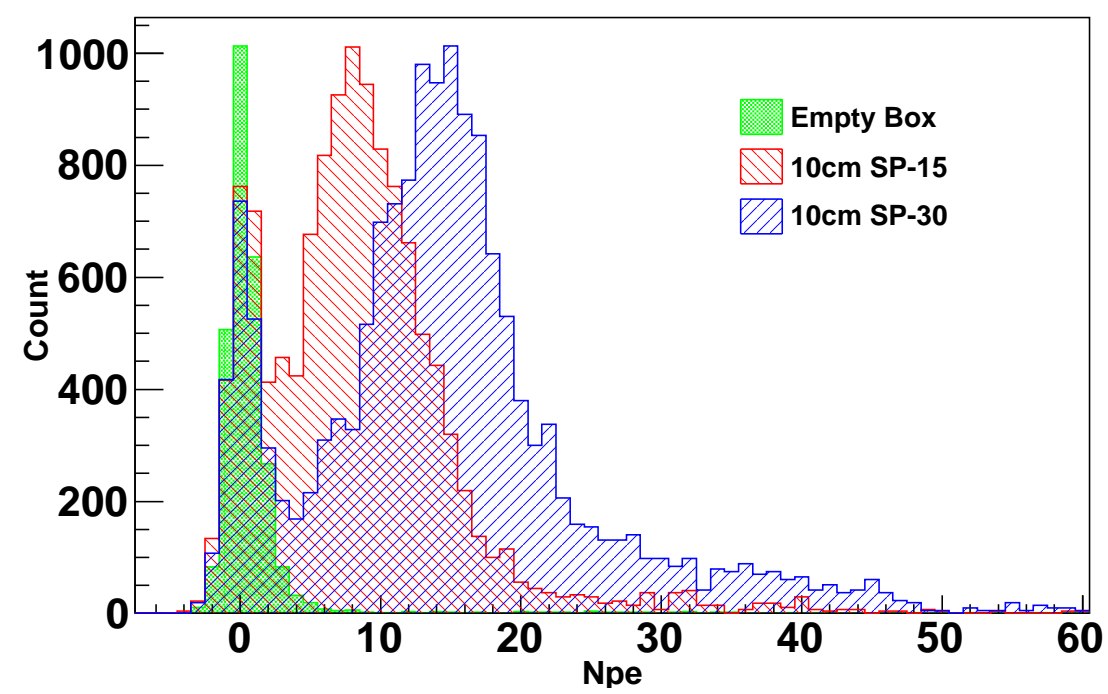

Figure 30: (Color online) The summed detector PMT signals from cosmic rays passing through a $10 \mathrm{~cm}$ stack of SP-15 (red) and SP-30 aerogel (blue) positioned at the center of the aerogel box. The green shaded area corresponds to the rays passing through an empty region outside the aerogel radiator, within the detector. The histograms are normalized to a common maximum height.

The above case somewhat resembles the single counter prototype discussed in section 3 . The difference in light absorption of the full diffusion box as compared to the single counter prototype was tested by next loading the detector box with different volumes of the aerogel material. Fig. 31 illustrates the detector response for a $10 \mathrm{~cm}$ thick single stack and a $2 \times 3$ aerogel stack. The data suggest a signal reduction of $\sim 30 \%$ when the aerogel volume is increased 6 -fold, in a matrix of two by three vertical aerogel stacks. 


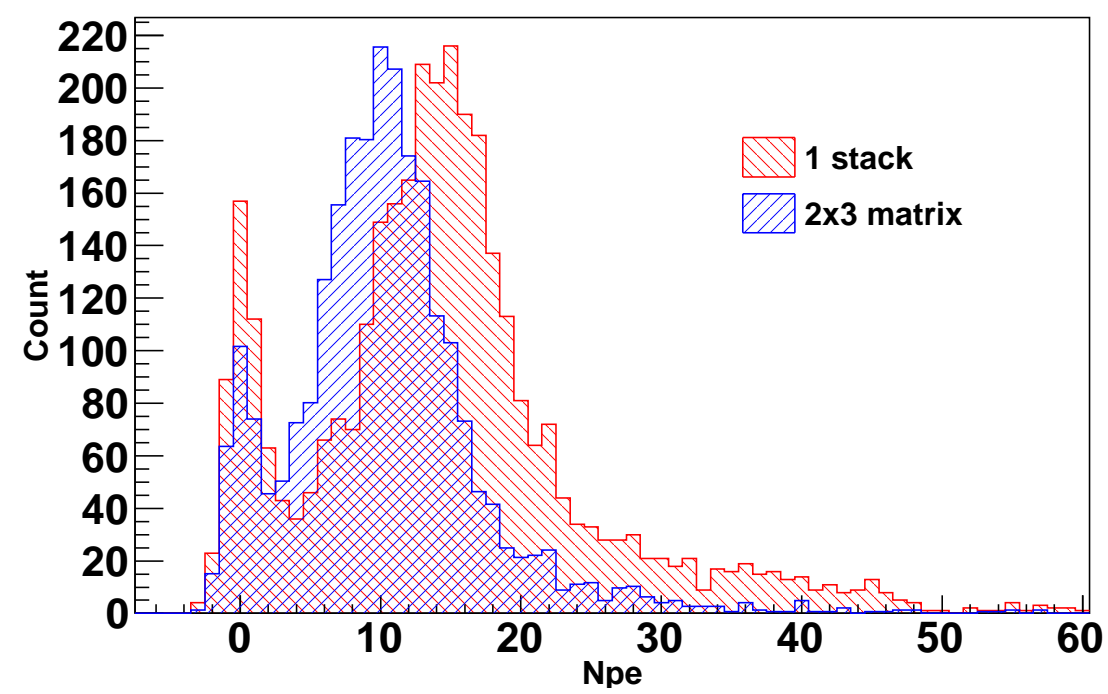

Figure 31: (Color online) Response of the detector to the cosmic rays passing through a 10 $\mathrm{cm}$ thick single stack of aerogel with refractive index $n=1.030$ at the center of the aerogel box (red), and through a matrix of $2 \times 3$ aerogel stacks (blue). The histograms are normalized to a common maximum height.

Naively one may assume that increasing the aerogel material volume will always yield more photoelectrons, and thus increase the detection efficiency. However, increasing the aerogel also increases the scattering and attenuation by absorption of photons. This was already observed in section 3.3 for the case of the single counter prototype, where the signal was shown to saturate with increasing aerogel thickness. This is as expected, due to scattering and absorption of the light by aerogel at short wavelengths.

Thus, finding the optimal aerogel material thickness is a balance at which one tries to keep the effect of light attenuation at a minimum, maximize the radiation of light, and reduce the amount of aerogel to minimize the production of $\delta$-electrons in it. After this threshold, the effects of attenuation begin to out-weight the gain of additional photoelectrons. To test the performance of the aerogel radiator in the full Cerenkov detector as a function of radiator

thickness, we carried out measurements with cosmic rays after installing every 
other aerogel layer during the tray assembly. Fig. 32 shows the results for aerogel with nominal refractive index $n=1.030$. Note that during these test measurements the aerogel detector was lined with Millipore reflector and was equipped with XP4500 PMTs. In the simulation results, the PMT quantum efficiencies were also degraded by a factor of 0.61 in accordance with the findings in our PMT studies (see section 4). The measurements are in agreement with the projections from the simulations.

At the optimal thickness of 8 layers $(8.8 \mathrm{~cm})$ of SP-30 aerogel, the summed signal from cosmic rays passing through the center of the detector has a peak value of $\sim 7$ photoelectrons. From here on, all further tests are performed with the aerogel Čerenlov detector diffusion box equipped with XP4572 PMTs and lined with a combination of $3.2 \mathrm{~mm}$ thick and $1.0 \mathrm{~mm}$ thick Gore reflective material, as described above, which raises the observed photoelectrons in this case to $\sim 11$ photoelectrons. 


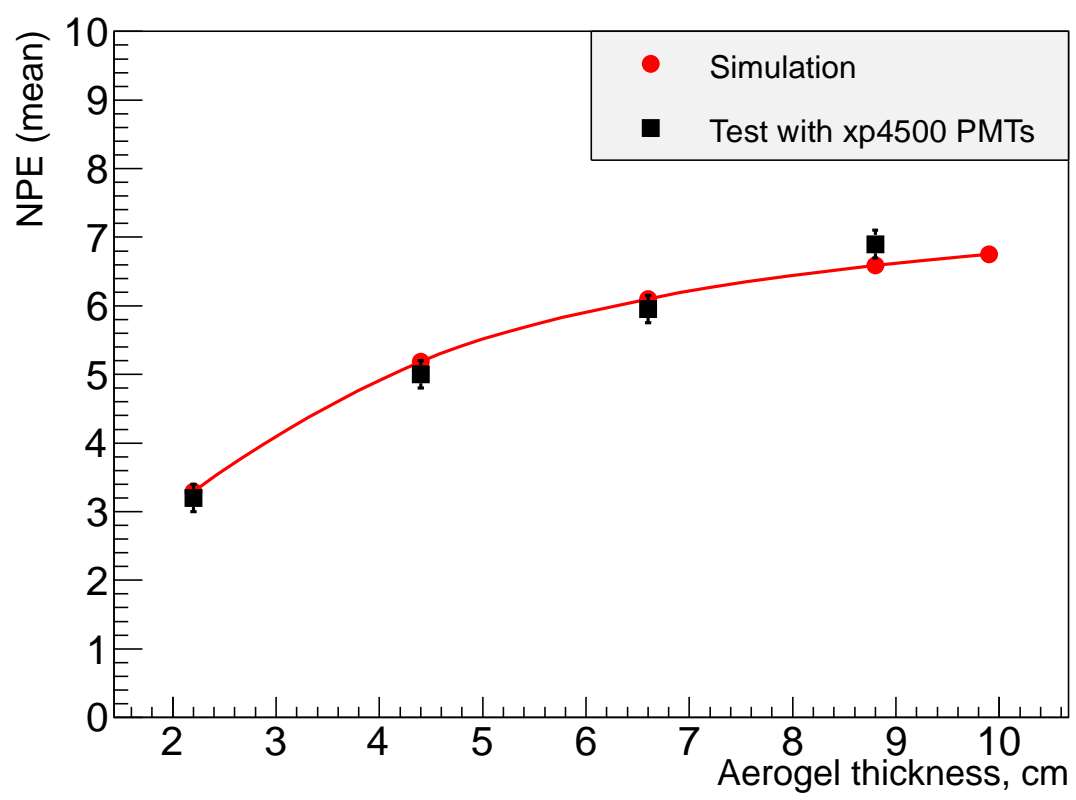

Figure 32: (Color online) The summed detector PMT signals from cosmic rays passing through the center of the detector as a function of the aerogel thickness. The data were obtained while filling the aerogel tray with aerogel with refractive index $n=1.030$ (SP-30). The solid squares indicate the measurements. The solid circles, and the line, denote the projections from the Monte Carlo simulations and the best second-order polynomial fit, and are in excellent agreement with the measurements. Note that the number of photoelectrons increases to $\sim 11$ (for an aerogel thickness of $8.8 \mathrm{~cm}$ ) if using XP4572 PMTs and lining of the aerogel diffusion box with Gore reflective material, see text.

Finally, we checked the spatial uniformity of the detector response by measuring the coordinate dependence of the detector signals, always with trays filled with 8 layers of aerogel $(8.8 \mathrm{~cm}$ thickness of aerogel material). For these tests the trigger scintillators were moved along two median lines: a horizontal scan from the left middle PMT to the right middle PMT, and a vertical scan from top to bottom along the line in the middle of the left and right PMTs of the detector. For each scan, the $\sim 3 "(\sim 8 \mathrm{~cm})$ wide and $\sim 16 "(\sim 40 \mathrm{~cm})$ long trigger scintillators were oriented transversely to the scan directions in order to minimize uncertainty in position. 
The vertical scans did not elucidate much: they do not show significant coordinate dependence of the signal, similar to the earlier observations with the HMS aerogel detector [16]. Figure 33 shows as an example the results from the vertical scan of the SP-30 and SP-20 trays, with the signals normalized at the center $(\mathrm{Y}=0 \mathrm{~cm})$.

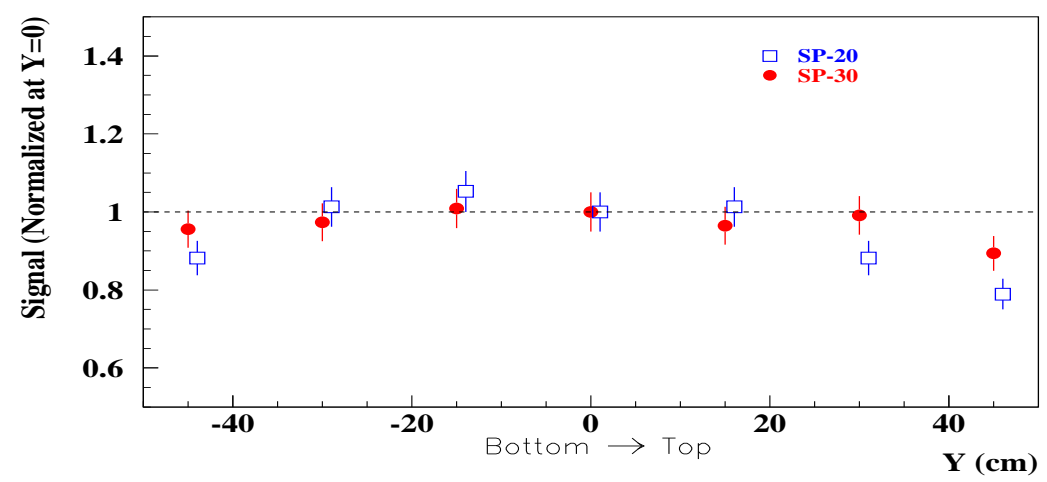

Figure 33: (Color online) Dependence of the summed signal from the SHMS aerogel detector on the impact point position of cosmic rays on the top - bottom median line of the detector. Signals from the detector with tray SP-30 and SP-20 are normalized at $\mathrm{Y}=0.0 \mathrm{~cm}$.

The horizontal scan for the aerogel with nominal refractive index $n=1.030$ is shown in Fig. 34. Recall that in this case the aerogel tray is lined with Millipore. The data show a summed signal of at least 11 photoelectrons at the center and an increase of the signal to above 12 photoelectrons at either edges of the detector, close to PMTs. This is not unexpected, the same behavior was observed for the HMS aerogel detector [16]. A slight asymmetry between left (positive $x$ ) and right (negative $x$ ) can be seen, more enhanced in the individual PMT sides (triangles and solid squares, for left and right PMTs, respectively). This asymmetry is likely caused by an imbalance of the quantum efficiency of the left and the right side PMTs. The simulated results, exhibited by the lines, do not show this left-right imbalance as an average quantum efficiency is assumed.

A similar dependence on the lateral coordinate was obtained for the aerogel 


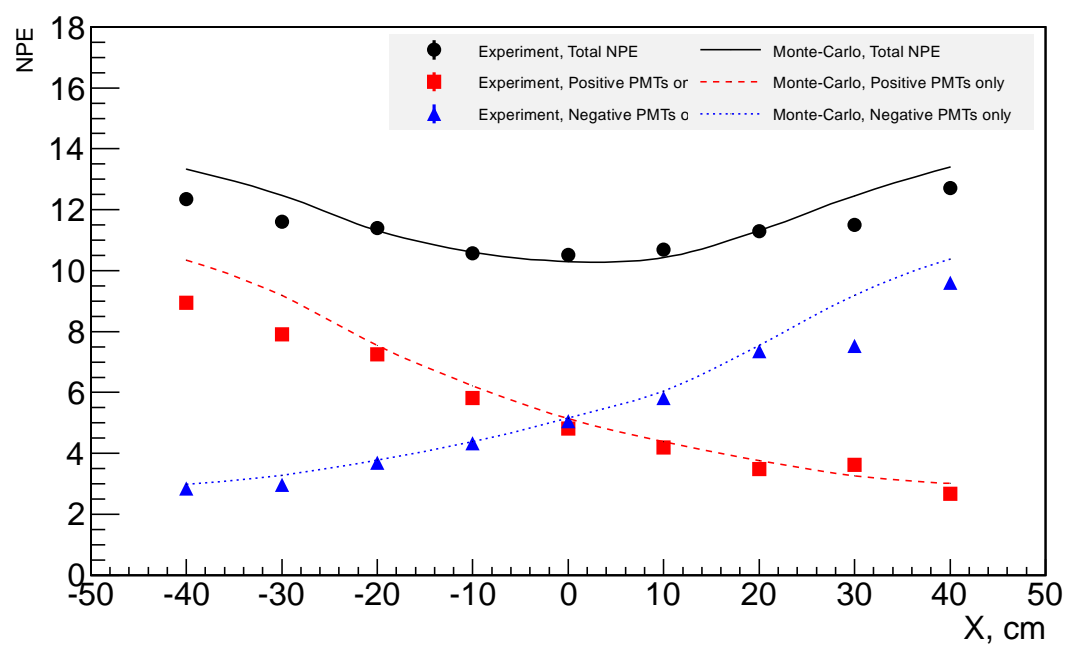

Figure 34: (Color online) Dependence of signals from the SHMS aerogel detector on the impact point position of cosmic rays on the left - right median line of the detector (see text). The tray is filled with $8.8 \mathrm{~cm}$ thick SP-30 type aerogel and lined with Millipore.

detector when using the aerogel trays as filled with refractive index $n=1.020$ and 1.015, SP-20 (Fig. 35) and SP-15 (Fig. 36), respectively. The data for SP20 show a signal amplitude of $\sim 7$ photoelectrons in the center, consistent in magnitude but slightly below what one would expect based on the amplitude measured with SP-30 aerogel, as illustrated by the comparison with the predictions from simulations (solid lines). An increase to above 8 photoelectrons can be observed at the right and the left edge of the detector. For the SP-20 case, the performance overall is below the expectation based on the simulations.

The SP-15 data show a signal amplitude of $\sim 10$ photoelectrons at the center of the detector. This is much higher than what one would naively expect from comparing to the results of the SP-30 and SP-20 tests. The SP-15 aerogel tray is lined with $1 \mathrm{~mm}$ Gore diffuse reflector material which may have increased the signal, but this can not account for all the increase noticed. The simulations shown in addition assume an aerogel absorption length of an order of magnitude higher than the $90 \mathrm{~cm}$ used for the SP-30 and SP-20 simulations. 


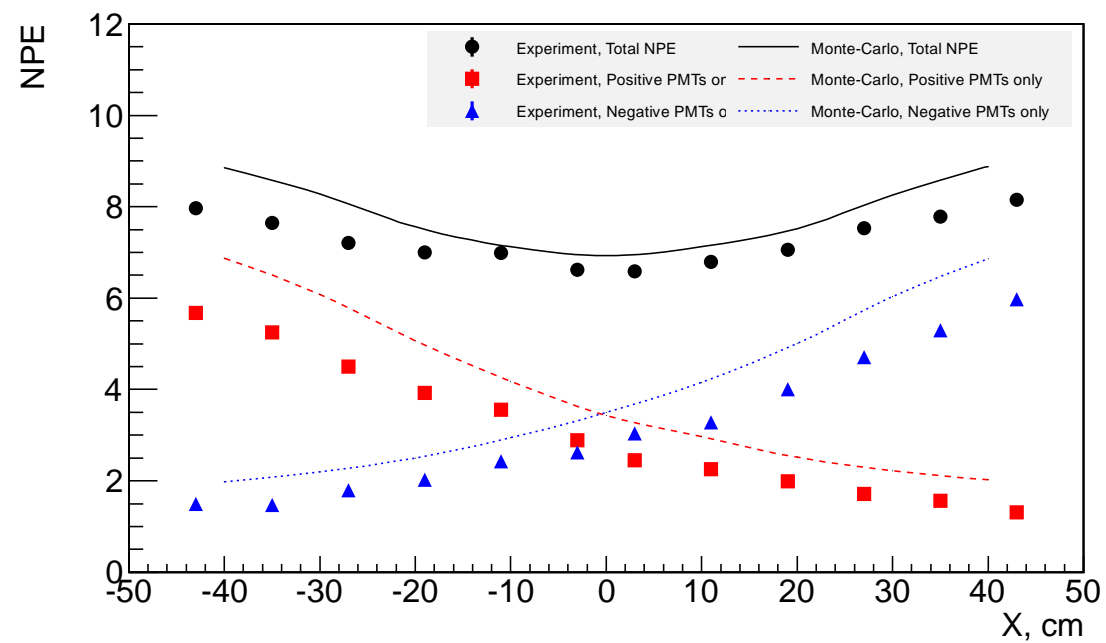

Figure 35: (Color online) Dependence of signals from the SHMS aerogel detector on the impact point position of cosmic rays on the left - right median line of the detector (see text). The tray is filled with $8.8 \mathrm{~cm}$ thick SP-20 type aerogel and lined with Millipore.

Note that the SP-15 and SP-11 aerogels were manufactured by a different manufacturer (Japanese Fine Ceramics Center) than the older SP-20 and SP-30 aerogels, possibly using a different production method. The larger signal amplitude may thus be partially due to improved quality of the aerogel material, as observed by our measurements of high absorption lengths (low light absorption) for the new aerogel. Again, an increase in signal closer to the PMTs can be seen, from $\sim 11$ photoelectrons on the right (positive PMT) up to $\sim 12$ photoelectrons on the left (negative PMT) 


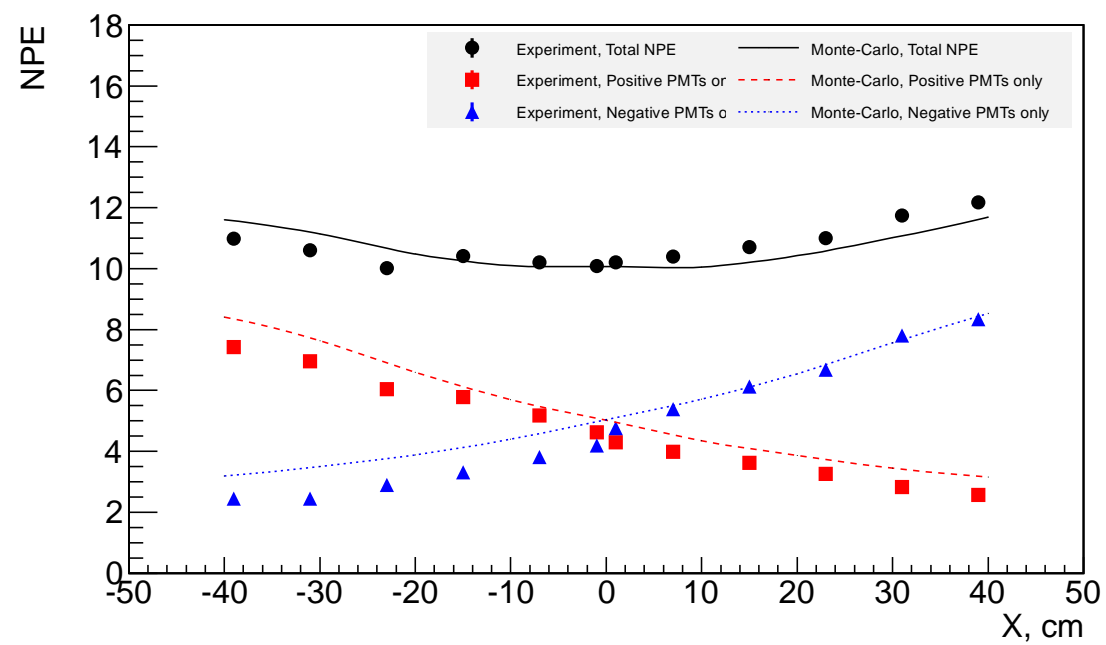

Figure 36: (Color online) Dependence of signals from the SHMS aerogel detector on the impact point position of cosmic rays on the left - right median line of the detector (see text). The tray is filled with $8.8 \mathrm{~cm}$ thick SP-15 type aerogel and lined with $1 \mathrm{~mm}$ Gore reflective material.

In general, if a constant threshold on the signal is used, a coordinate dependence of the summed signal impacts both detection efficiency and rejection capability in the experimental data analysis. To address this issue and to optimize the detector's performance the threshold level can be adjusted to be optimal for tracks at the center of the acceptance. Alternatively, one could vary the threshold depending on the coordinate, and ensure uniform performance across the acceptance.

A comparative study of the light yield performance of the different aerogel refractive indices is shown in Fig. 37. The measurements were carried out with cosmic rays using the single counter described in section 3 and the full detector. For this specific test, the single counter was covered with Aluminized Mylar. The full detector trays were covered with Millipore (SP-30 and SP-20) and $1 \mathrm{~mm}$ thick GORE (SP-15, SP-11) reflector material. The diffusion box was covered with 60 (40)\% 3.2 (1.1) $\mathrm{mm}$ thick GORE reflective material. The data from the single counter measurements are well described by a fit of the form of 
Eq. 3. The light yield from the full detector is higher by about a factor of two for 980 the higher two refractive indices. This is expected since the reflector material used for covering the interior of the full detector was optimized. Based on Fig. 25, the reflectivity of aluminized mylar is about $85 \%$ and that of Millipore about $95 \%$ around $400 \mathrm{~nm}$, where the PMTs are most sensitive. Assuming ten interactions of a photon with the detector walls, this would result in light loss 985 of $80 \%$ with mylar compared to $40 \%$ with Millipore reflector. The point at refractive index $n=1.015$ shows a $60 \%$ higher light yield since the SP-15 tray was optimized with GORE reflector, which has an even higher reflectivity than Millipore. Taking into account the enhancement due to the higher reflectivity of GORE and Millipore to mylar as shown in Fig. 25, the light yield data follow the expected trend as a function of refractive index. 


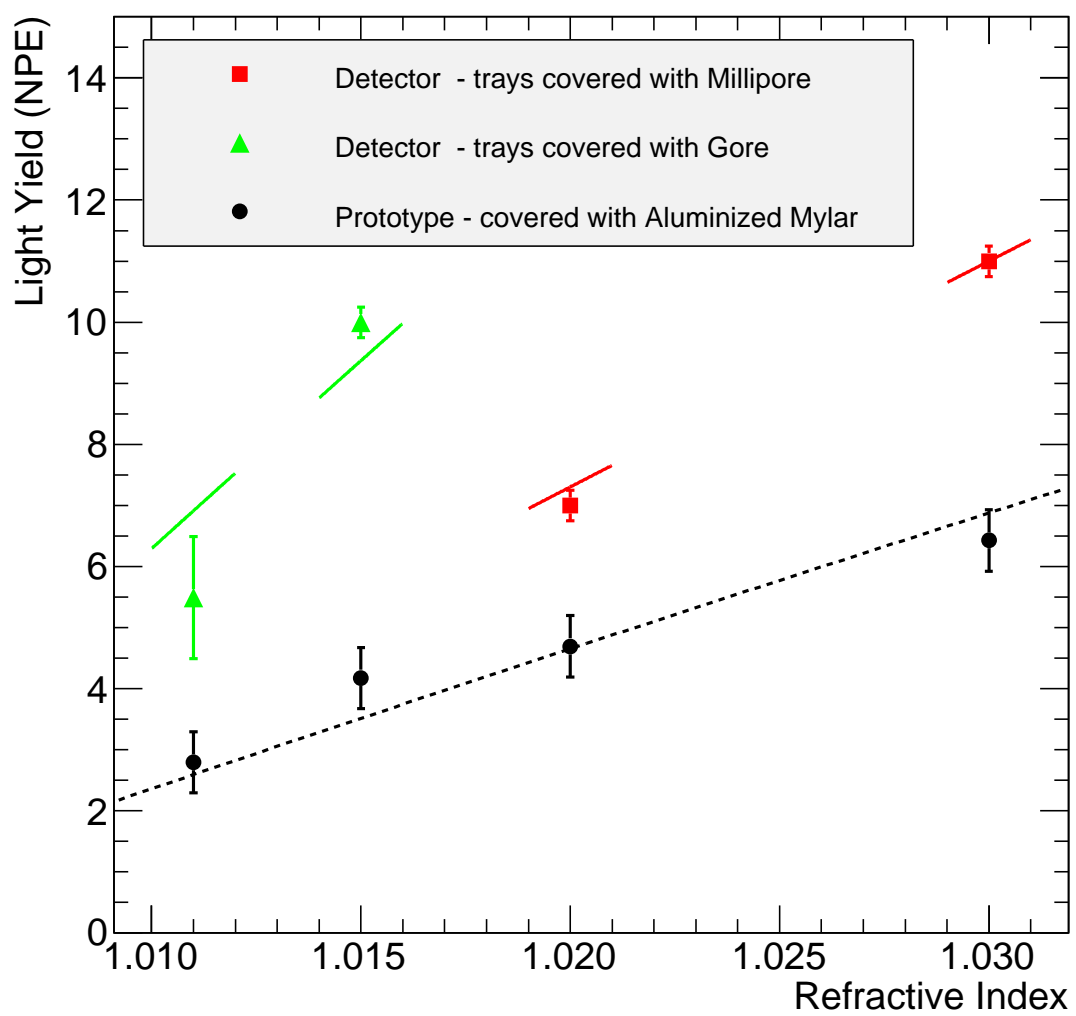

Figure 37: (Color online) Comparative study of the light yield of all four aerogel refractive indices. Aluminized mylar was used to cover the internal walls of the prototype, while Millipore covers the trays with refractive index $n=1.030$ and $n=1.020$ and Gore reflector covers the lowers index trays. The solid lines denote the expected increase in light yield measured with the full detector compared to the single counter owing predominantly to increased reflectivity of the material covering the interior walls. See text for details.

\section{SUMMARY}

In summary, we have constructed a threshold Aerogel Čerenkov detector with nominal refractive indices $n=1.030,1.020,1.015$, and 1.011 , that can be used in the SHMS magnetic spectrometer in Hall $\mathrm{C}$ at JLab for experiments requiring kaon particle identification. The detector consists of one diffusion 
box and four exchangeable aerogel trays each loaded with a different nominal refractive index.

The aerogel material of the higher refractive indices $(n=1.030$ and $n=1.020)$ was originally manufactured by Matsushita and used in the BLAST experiment. as index of refraction, transmittance and light yield was studied in detail. We found that these aerogel materials are generally of very good quality and there is no evidence for quality changes of the previously used material.

The photosensors were also characterized carefully, in particular their gain and quantum efficiency. The Photonis PMTs model XP4500 has a high gain, but shows a $\sim 30-40 \%$ lower quantum efficiency than the flat-faced PMTs model XP4572. For the final assembly 14 PMTs (XP4572) were selected from a pool 25 five-inch diameter PMTs based on the criteria of high quantum efficiency, low noise, high gain at relatively low high voltage, and good single photoelectron resolution.

The light collection performance of the detector was tested with cosmic rays and proton beams. The detector signal shows good uniformity along the vertical (Y) coordinate of the detector surface, but has a significant dependence in the horizontal $(\mathrm{X})$ direction. Possible optimization of this include a variable threshold and an optimized selection of the PMTs installed on the right and left side of the detector.

The mean number of photo-electrons in saturation for the tray filled with $n=1.030(n=1.020)$ refractive index aerogel is $\sim 12(\sim 8)$ which is close to MC simulation. For the trays filled with $n=1.015$ and $n=1.011$ refractive index aerogel, high number of photoelectrons were obtained with the use of higher reflectivity material to cover the tray, $\sim 10$ and $\sim 5.5$ respectively. This result could be fully reproduced by our Monte Carlo simulation by also assuming the aerogel absorption length on the order of $220 \mathrm{~cm}$.

These results were obtained using Gore diffusive reflector material and optimizing the configuration of PMTs installed on either side of the detector. 
Using a lining of the Čerenkov aerogel detector of Gore diffusive reflector (with reflectivity above 99\%) rather than the more commonly used Millipore (with reflectivity of $96 \%$ ) drastically improved the performance of the detector, by $1030 \sim 35 \%$.

\section{ACKNOWLEDGMENTS}

This work was supported in part by NSF grants PHY-1039446 and PHY1306227, the CUA physics department and the Vitreous State Laboratory (VSL). In particular, the authors wish to thank Marek Brandys, Eric Fisher, and David Horton from the VSL for their expertise and support in the construction of the detector. The detector benefited greatly from components graciously provided by both the MIT-BLAST collaboration (aerogel materials and PMTs) and Hall C (PMTs). We explicitly are greatful to Ricardo Alarcon and Richard Milner for help provided to acquire and transport the MIT-BLAST aerogel detector components. We would also like to thank Carl Zorn from the Detector Group of the Jefferson Lab Physics Division, the Hall C engineering staff, in particular Bert Metzger for help with the design of the detector and expertise during assembly, and Walter Kellner and the Hall C technical staff, Joe Beaufait, and Brad Sawatzky, as well as Mariana Khachatryan from ANSL for help during various stages of the work. The Southeastern Universities Research Association operates the Thomas Jefferson National Accelerator Facility under the U.S. Department of Energy contract DEAC05-84ER40150.

\section{References}

[1] N. S. A. Committee, "the 2015 long range plan for nuclear science", http://science.energy.gov/ /media/np/nsac/pdf/2015LRP/2015_LRPNS_091815.pdf (2015).

[2] R. D. McKeown, Jefferson Lab 12 GeV Science Program, Int. J. Mod. Phys. Conf. Ser. 37 (2015) 1560019. doi:10.1142/S2010194515600198. 
[3] J. Dudek, et al., Physics Opportunities with the $12 \mathrm{GeV}$ Upgrade at Jefferson Lab, Eur. Phys. J. A48 (2012) 187. arXiv:1208.1244, doi:10.1140/epja/i2012-12187-1

[4] H. P. Blok, et al., Charged pion form factor between $Q^{2}=0.60$ and $2.45 \mathrm{GeV}^{2}$. I. Measurements of the cross section for the ${ }^{1} \mathrm{H}\left(e, e^{\prime} \pi^{+}\right) n$ reaction, Phys. Rev. C78 (2008) 045202. arXiv:0809.3161, doi:10.1103/PhysRevC.78.045202

[5] S. Lassiter, P. Brindza, E. Sun, M. Fowler, G. Dickson, The analysis and measurement of composite coil properties of Jefferson Lab's super high momentum spectrometer (SHMS) superconducting magnet coils, AIP Conf. Proc. 1218 (2010) 965-972. doi:10.1063/1.3422467.

[6] Horn T., Huber G.M., and others, "scaling study of the l-t separated pion electroproduction cross section at 11 gev", approved Jefferson Lab $12 \mathrm{GeV}$ Experiment (2007).

[7] Horn T., Huber G.M., Markowitz P., and others, "studies of the l-t separated kaon electroproduction cross section from 5-11 gev", approved Jefferson Lab $12 \mathrm{GeV}$ Experiment (2009).

[8] T. Horn, C. D. Roberts, The pion: an enigma within the Standard Model, J. Phys. G43 (7) (2016) 073001. arXiv:1602.04016, doi:10.1088/0954-3899/43/7/073001.

[9] L. Favart, M. Guidal, T. Horn, P. Kroll, Deeply Virtual Meson Production on the nucleon, Eur. Phys. J. A52 (6) (2016) 158. arXiv:1511.04535, doi:10.1140/epja/i2016-16158-2

[10] Bosted P., Ent R., Kinney E., Mkrtchyan, H., and others, "transverse momentum dependence of semi-inclusive pion production", approved Jefferson Lab $12 \mathrm{GeV}$ Experiment (2009). 
[11] Bosted P., Ent R., Kinney E., Mkrtchyan, H., and others, "measurement of the ratio $r=\sigma_{l} / \sigma_{t}$ in semi-inclusive deep-inelastic regimes", approved Jefferson Lab 12 GeV Experiment (2006).

[12] Dutta D., Ent R., and others, "the search for color transparency at 12 gev", approved Jefferson Lab 12 GeV Experiment (2006).

[13] D. W. Higinbotham, Diffusely reflective aerogel Cherenkov detector simulation techniques, Nucl. Instrum. Meth. A414 (1998) 332-339. doi:10.1016/S0168-9002(98)00692-5

[14] M. Benot, et al., Tests of Large Cherenkov Detectors With Silica Aerogel as Radiator, Nucl. Instrum. Meth. 154 (1978) 253. doi:10.1016/0029-554X(78) 90407-X.

[15] J. Alcorn, et al., Basic Instrumentation for Hall A at Jefferson Lab, Nucl. Instrum. Meth. A522 (2004) 294-346. doi:10.1016/j.nima.2003.11.415

[16] R. Asaturyan, et al., The aerogel threshold Cerenkov detector for the high momentum spectrometer in Hall $\mathrm{C}$ at Jefferson Lab, Nucl. Instrum. Meth. A548 (2005) 364-374. arXiv:physics/0411147, doi:10.1016/j.nima.2005.04.058

[17] G. Poelz, AEROGEL CHERENKOV COUNTERS AT DESY, Nucl. Instrum. Meth. A248 (1986) 118. doi:10.1016/0168-9002(86)90504-8.

[18] P. Carlson, Aerogel Cherenkov Counters: Construction Principles and Applications, Nucl. Instrum. Meth. A248 (1986) 110-117. 1100 doi:10.1016/0168-9002(86)90503-6.

[19] Gore, W. L. \& Assosiates INC., "”, http://www.gore.com.

[20] Millipore Corporation, 80 Ashly Road, Bedford, MA 01730, "”, http://www.millipore.com/. 
[21] I. Adachi, et al., Study of highly transparent silica aerogel as a RICH radiator, Nucl. Instrum. Meth. A553 (2005) 146-151. doi:10.1016/j.nima.2005.08.022

[22] E. Aschenauer, et al., Optical characterization of $n=1.03$ silica aerogel used as radiator in the RICH of HERMES, Nucl. Instrum. Meth. A440 (2000) 338-347. doi:10.1016/S0168-9002(99)00923-7.

[29] T. Sumiyoshi, et al., Silica aerogel Cherenkov counter for the KEK B factory experiment, Nucl. Instrum. Meth. A433 (1999) 385-391. doi:10.1016/S0168-9002(99)00460-X.

[30] A. F. Danilyuk, V. L. Kirillov, M. D. Saveleva, V. S. Bobrovnikov, A. R. Buzykaev, E. A. Kravchenko, A. V. Lavrov, A. P. Onuchin, Recent results 
[37] Coman, M., "the hall a aerogel Čerenkov detector", master Thesis, FIU (2000).

[38] J. E. Grove, R. A. Mewaldt, The Contribution to Cherenkov radiation from knockon electrons, Nucl. Instrum. Meth. A314 (1992) 495-503.

on aerogel development for use in Cherenkov counters, Nucl. Instrum. Meth. A494 (2002) 491-494. doi:10.1016/S0168-9002(02)01537-1.

[31] Barnyakov, A. Yu., "aerogel cherenkov counter for the snd detector", sND Symposium, Stanford, California (2006).

[32] D. Androic, et al., The G0 Experiment: Apparatus for ParityViolating Electron Scattering Measurements at Forward and Backward Angles, Nucl. Instrum. Meth. A646 (2011) 59-86. arXiv:1103.0761, doi:10.1016/j.nima.2011.04.031

[33] ElJen Technology, 1300 W Broodway, Sweetwater, TX 79556, USA, "”, www.eljentechnology.com.

[34] K. Allada, C. Hurlbut, L. Ou, B. Schmookler, A. Shahinyan, B. Wojtsekhowski, PMT signal increase using a wavelength shifting paint, Nucl. Instrum. Meth. A782 (2015) 87-91. arXiv:1502.01772, doi:10.1016/j.nima.2015.02.012

[35] T. Gogami, et al., Bucking coil implementation on PMT for active canceling of magnetic field, Nucl. Instrum. Meth. A729 (2013) 816-824. arXiv:1307.0896, doi:10.1016/j.nima.2013.08.047.

[36] S. Nishida, I. Adachi, T. Iijima, H. Ikeda, S. Korpar, P. Krizan, Y. Miyazawa, I. Nishizawa, T. Sumiyoshi, Development of an HAPD with 144 channels for the aerogel RICH of the Belle upgrade, Nucl. Instrum. Meth. A595 (2008) 150-153. doi:10.1016/j.nima.2008.07.032. doi:10.1016/0168-9002(92)90240-5 
[39] Berger, M. J. and Seltzer, S. M., "nasa technical report sp-3012", nASA Technical Report (1964).

[40] Berger, M. J. and Seltzer, S. M., "”, 14th International Cosmic Ray Conference, Conference Papers, vol. 9, p.3177, Munich (1975).

[41] H. Mkrtchyan, et al., The lead-glass electromagnetic calorimeters for the magnetic spectrometers in Hall $\mathrm{C}$ at Jefferson Lab, Nucl. Instrum. Meth. A719 (2013) 85-100. arXiv:1204.6413, doi:10.1016/j.nima.2013.03.070

[42] , "”, http://geant4.cern.ch/support/proc-mod-catalog/physics-lists.

[43] L. W. Hrubesh, OPTICAL CHARACTERIZATION OF SILICA AEROGEL GLASS.

[44] Photomultipliers, Philips, PC04,"”, http://www.photonis.com/upload/industryscience/pdf.

\section{Appendix}

\subsection{Details on the Monte Carlo Simulation of the Detector}

The program for simulation of the SHMS aerogel detector is based on the GEANT4 package [42], version 9.2. It includes sampling of incoming particles at the focal plane of the spectrometer and passing them through the material of the detectors before the aerogel counter; Cerenkov light generation in the aerogel and its propagation up to the PMT photocathodes; and signal generation from the PMTs. Interaction of the particles with materials is modeled within the framework of the Quark-Gluon String Precompound physics model (QGSP), and the standard GEANT4 optics model is used for the light generation and propagation.

The total thickness of the material from the SHMS detectors located between spectrometer magneto-optical focal plane and the aerogel Čeerenkov detector amounts to $3.7 \mathrm{~g} / \mathrm{cm}^{2}$ (see Table 5 ). The front wall of the aerogel detector adds 
Table 5: The materials between the SHMS focal plane and the aerogel Čerenkov detector. The listed positions are at the fronts of components, as measured from the central point (particle momentum equals the set spectrometer momentum) magneto-optical focal plane.

\begin{tabular}{|c|c|c|c|}
\hline component & material & $\begin{array}{c}\text { position } \\
(\mathrm{cm})\end{array}$ & $\begin{array}{c}\text { thickness } \\
\left(\mathrm{g} / \mathrm{cm}^{2}\right)\end{array}$ \\
\hline DC2 gas & Ethane/Ar & 40 & 0.005 \\
DC2 foils & Mylar & & 0.025 \\
S1X hodoscope & BC408 scint. & 50 & 0.516 \\
S1Y hodoscope & BC408 scint. & 60 & 0.516 \\
Gas C - entr. window & Al & 80 & 0.270 \\
Gas Č - gas & $C_{4} F_{8} O$ & & 0.975 \\
Gas C - mirror & glass & & 0.720 \\
Gas C - mirror support & carbon fiber & & 0.180 \\
Gas Č - exit window & Al & 180 & 0.270 \\
Air & Air at STP & & 0.181 \\
\hline Total & & & 3.658 \\
\hline
\end{tabular}

$\sim 0.2 \mathrm{~g} / \mathrm{cm}^{2}$. The density of the aerogel is derived from refractive index $n$ using $(n-1) / 0.21\left(\mathrm{~g} / \mathrm{cm}^{3}\right)$ 25]. For example, $9 \mathrm{~cm}$ of $n=1.030$ aerogel material would amount to an effective thickness of $\sim 1.3 \mathrm{~g} / \mathrm{cm}^{2}$.

The aerogel material is characterized by a constant refractive index, and by wavelength-dependent absorption and scattering lengths, $\Lambda_{A}$ and $\Lambda_{S}$. The last 2 parameters were modeled in the simulation based on studies of aerogel optical properties carried out at DESY [22]. For light of wavelengths $\lambda>300$ $\mathrm{nm}$ the transmittance through the aerogel is described to good accuracy by the Hunt formula [43] which assumes constant absorption (constant $\Lambda_{A}$ ) and Rayleigh scattering $\left(\Lambda_{S} \sim \lambda^{4}\right)$. Below $300 \mathrm{~nm} \Lambda_{A}$ dramatically decreases, and $\Lambda_{S}$ changes less rapidly.

Absorption and scattering lengths of the aerogel used in the simulations 
include a constant absorption length for $\lambda>300 \mathrm{~nm}$ linearly decreasing to 1 $\mathrm{cm}$ at $\lambda \approx 200 \mathrm{~nm}$ in accordance with Ref. [22]. The constant value for $\lambda>$ $300 \mathrm{~nm}$ was tuned using data from cosmic tests with the SHMS aerogel Čerenov detector (see section 5.3.3) and optimized for the optimum sensitivity of our PMTs, around $450 \mathrm{~nm}$. A value of $\Lambda_{A}=90 \mathrm{~cm}$ gives the best agreement between simulation and data for SP-30 and SP-20 aerogels. This value is also consistent with our direct measurement of the absorption length at $450 \mathrm{~nm}$ as shown in Fig. 13. For the lower refractive index aerogels, which were produced by a different vendor, a larger value seems to be more suitable, we find $\Lambda_{A} \sim 220 \mathrm{~cm}$. The scale of $\Lambda_{S}$ is of less importance for the simulation accuracy of the detector's performance. For the purpose of the present simulations it was derived from the average value for the Hunt clarity factor $C t$ obtained in Ref. [22].

Reflectivity of the Millipore paper for $\lambda \geq 315 \mathrm{~nm}$ is taken from [14]. Below $315 \mathrm{~nm}$ it is linearly extrapolated to $50 \%$ at $\lambda=190 \mathrm{~nm}$. The Millipore reflectance is taken pure $(100 \%)$ Lambertian. The reflectance of Gore material is taken from Ref. [19].

The geometry of the PMT spherical window together with adjacent photocathode is coded using dimensions of the XP4500 and XP4572 PMT from the vendor [44]. The mean quantum efficiency of the bialcali photocathode is derived from typical spectrum of radiant sensitivity, also from the vendor. 